\title{
5 Schule Breitenrain in der Stadt Bern - Von der Disziplinierung zur intrinsischen Selbstdisziplin
}

Das Schulhaus Breitenrain in der Stadt Bern verkörpert prototypisch den damaligen Gebäudetypus Schulhaus, der im Volksmund Schulkaserne genannt wurde. Das Gebäude ist nach Strukturprinzipien errichtet worden, die für die damalige Schule typisch sind und die bis heute Bestand haben. In diesem Sinne steht die exemplarische Analyse des Schulhauses Breitenrain für die Genese, Konsolidierung und auch die Brüche des staatlichen Schulsystems, wie sie sich in den Schulgebäuden objektivieren.

\subsection{Die Bauhistorie des Schulhauses Breitenrain}

Das Schulhaus Breitenrain wurde 1865 vom Architekten Carl Haller erbaut (damals noch Schulhaus Lorraine genannt). ${ }^{23}$ Im Zeitverlauf kamen verschiedene Erweiterungsbauten hinzu, und es wurden diverse Umbauten und Sanierungen vorgenommen. Als erste Erweiterung wurde 1880 in einem rechten Winkel zum Schulhaus die Turnhalle erstellt. 1926 wurde hofseitig eine erste und 1936 eine zweite Toilettenanlage erstellt. Im gleichen Jahr wurde auch die Turnhalle um eine Garderobe erweitert. Zudem wurde der Durchgang zwischen Turnhalle und Schulhaus als Pausenhalle überdeckt. Mit Ausnahme der Turnhalle, die heute unter Denkmalschutz steht, wurden diese Gebäudeteile abgerissen. 2004 wurde die Turnhalle saniert und der Garderobenbau durch einen Neubau ersetzt. Im Jahr darauf wird das Schulhaus von der Rykart Architekten AG saniert und der Pausenhof neugestaltet.

23 Die Bauhistorie stellt methodisch gesehen einen Teil der objektiven Daten dar. Der Zweck ist, einen Überblick über das Handlungsproblem und die Situierung des Falls innerhalb der Rahmenbedingungen der Datenlage zu gewinnen. Auf die Darstellung der Interpretation der objektiven Daten wird aus Platzgründen verzichtet, doch wird am Ende des Abschnitts ein kurzes Zwischenergebnis als Skizze des Möglichkeitsraums formuliert, der durch die Bauhistorie gegeben ist. 
Das ursprüngliche Schulhaus bleibt bei allen Um- und Ausbauten in seiner maßgeblichen Gestalt bestehen. Dass das Hauptgebäude und die Turnhalle unter Denkmalschutz steht, bedeutet möglicherweise eine bauliche Bürde, wenn man berücksichtigt, dass alle anderen Anbauten abgerissen wurden und dass insbesondere die Turnhalle nicht mehr den heutigen Ansprüchen einer polysportiven Turnhalle genügt. Die Fallrekonstruktion beginnt mit der Rekonstruktion des Luftbilds (Abb. 4 und 5).

\subsection{Siedlungsbild Breitenrain/Lorraine in der Stadt Bern - Das Schulhaus als Repräsentationsbau von Staatlichkeit und Bevölkerungspolitik}

Im Bild sieht man ein städtisches Quartier, das geprägt ist durch Wohnhäuser, die größtenteils am Ende des 19. und im ersten Drittel des 20. Jahrhunderts entstanden sind. Das Quartier besteht aus Reihen aneinandergebauter Häuser, die teilweise als Gebäudegruppen einen Innenhof bilden. Die Häuser weisen relativ einheitliche Bauvolumen und Bauweisen auf. Die Bebauung ist recht dicht. Nur im Norden in der Mitte des Bildes befindet sich eine Zeile von kleineren und älteren Häusern mit jeweils zugehörigem Garten.

Die Straßenzüge und Häuser im südöstlichen Teil des Bildes sind leicht verschoben, von Nord-Nordost nach Süd-Südwest ausgerichtet. Auch aufgrund der Straßenzüge müssen hierfür topographische Gründe angenommen werden. Die Verkehrsführung gliedert den betrachteten Ausschnitt in drei Teile. Die Hauptachse, der Nordring ${ }^{24}$, verläuft gekrümmt in Nord-Süd-Richtung. Durch sie wird das angrenzende Stadtviertel, die ,Lorraine“ (links im Bild), abgetrennt. In der Mitte des Bildes kreuzt die zweitgrößte Verkehrsachse (Breitenrainstraße) den Nordring. Dabei legt die gekrümmte Häuserzeile nahe, dass die Verkehrsrichtung nicht die Kreuzung des Nordrings beinhaltet, sondern dass die Breitenrainstraße in den Nordring mündet. 


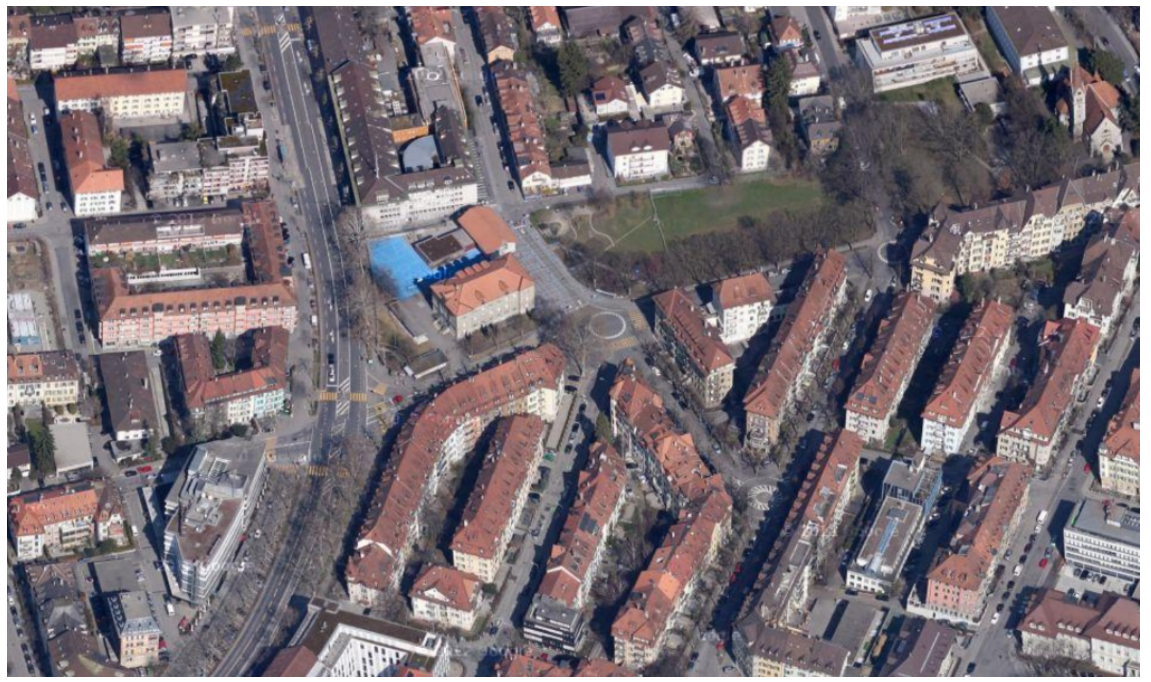

Abb. 4: Luftaufnahme Quartier Breitenrain/Lorraine (Stadt Bern). (C) Google Maps 2013

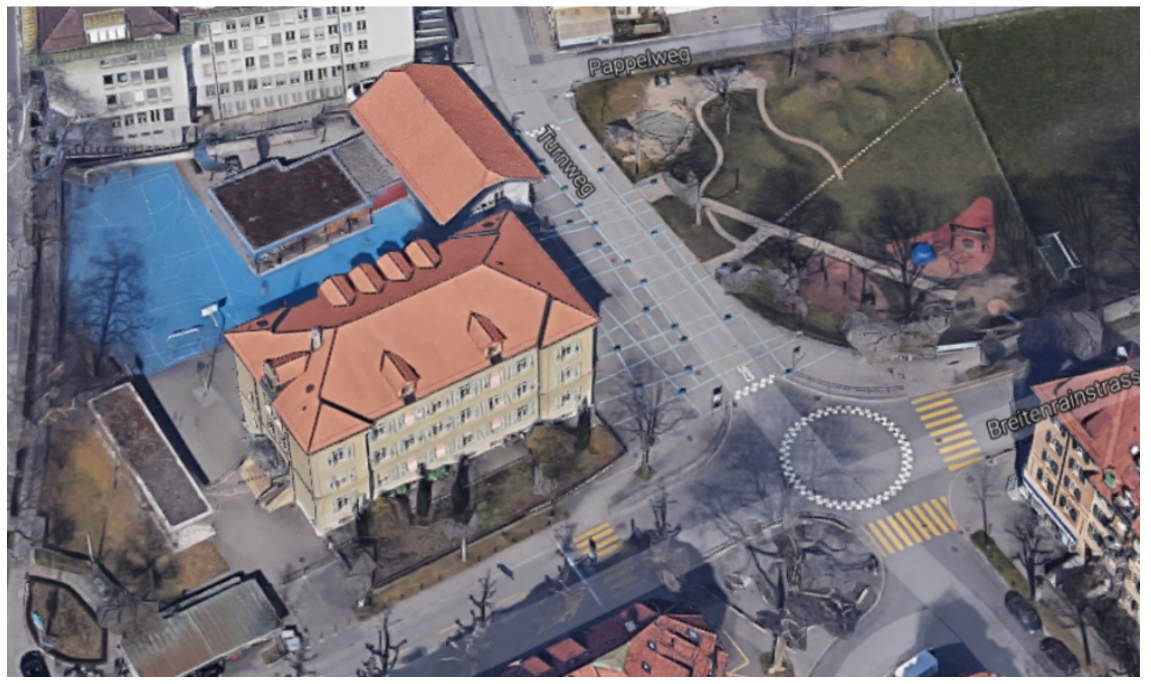

Abb. 5: Luftaufnahme Schulanlage Breitenrain. (C) Google Maps 2013 
Im Innenwinkel dieser Weggabelung befindet sich ein Areal, das sich von den umgebenden Wohnhäusern deutlich abhebt. Es scheint aus verschiedenen, funktional differenzierten Flächen und Gebäuden zu bestehen. Direkt an der Verzweigung befindet sich ein erster Perimeter mit insgesamt fünf unterschiedlichen Bauten, die sich in Größe und Form zwar stark unterscheiden, aber gerade dadurch eine Einheit zu bilden scheinen. Besonders herausstechend ist der blaue Platz. ${ }^{25}$

Abgesehen von der Farbigkeit ist es vor allem die Form eines konvexen Vierecks des Perimeters, die die Gebäudegruppe gegenüber den rationalisierten, rechtwinklig angelegten Reihungen der Umgebung abhebt. Auch sind die Gebäude anders ausgerichtet. Zudem zeigt sich in der geometrischen Anordnung eine Fortführung dieses konvexen Grundstücks nach rechts. In der Fortsetzung der Häuser und der rahmenden Straßenzüge zeichnet sich ein sowohl getrenntes als auch zugehöriges Gebiet mit Grünflächen und Baumbepflanzungen ab. Neben der Form wird der angrenzende Park gestalterisch mit dem Gelände durch die blauen Objekte und durch die Linien verbunden, die über die Straße in den Park führen (siehe Abb. 6). Die Rasenfläche ist in verschiedene Elemente unterteilt. Auf der Ostseite befindet sich ein großes abgetrenntes Stück Rasen, ein Fußballfeld. Auf der westlichen Seite ist eine mehrfach unterteilte Fläche zu sehen, die einen Spielplatz darstellt. Ein gepunkteter, mit Steinen belegter Weg führt geradlinig zu einem Tor im Zaun. Es sind unterschiedliche Bereiche erkennbar, die verschiedene Spielmöglichkeiten bieten. Die gesamte Rasenfläche ist an den Rändern von Bäumen umgeben. Das Gras weist unterschiedliche Farben auf, was auf einen unebenen Untergrund schließen lässt. Abgeschlossen wird das Gebiet im Osten von einer Straße und einer Kirche.

Aus der Lage geht hervor, dass sich der Gebäude- und Flächenkomplex an einem verkehrstechnischen Knotenpunkt befindet. Wie bereits ausgeführt kann von einem y-förmigen Verkehrsstrom beziehungsweise von einer Verzweigung von Nordring und Breitenrainstraße ausgegangen werden, bei welchem das Zent-

25 Methodisch könnte man auch umgekehrt vorgehen, wenn die Beschreibung dem ikonographischen Pfad folgte. Dann würde mit dem blauen Platz begonnen. An der Analyse der Sinnstrukturen würde sich damit nichts ändern. Die Schulanlage und der blaue Platz werden als Zentrum des Bildausschnittes rekonstruiert, um den herum sich sowohl die Verkehrswege als auch die Gebäudeausrichtungen ziehen. 
rum weiter südlich liegt. Der Gebäudekomplex liegt an einem städtebaulichen Knotenpunkt mit verkehrstechnischer Bedeutung.

Die Lage des Areals hat zur Folge, dass sich der Verkehr am Gebäude, das direkt an der Breitenrainstraße liegt, vorbeibewegt. Offensichtlich ist nicht das Gebäude der Zielpunkt der Verkehrsführung. Vielmehr positioniert es sich prominent an der stadtauswärtsführenden Verzweigung. ${ }^{26}$ Das Gebäude stellt einen markanten Punkt dar, an dem zwangsläufig vorbeikommt, wer sich auf den für den Durchgangsverkehr ausgelegten Straßen bewegt.

Diese zentrale Positionierung scheint nicht der Funktionalität geschuldet zu sein: Es sind keine Hinweise darauf erkennbar, dass das Haus auf ein großes Verkehrsaufkommen angewiesen ist, wie dies zum Beispiel bei einem Bahnhofsgebäude oder einer Tankstelle der Fall ist. Die Lage deutet eindeutig auf Repräsentationsabsichten hin. Das Gebäude soll von den Vorbeiziehenden gesehen werden und nimmt dadurch für sich ein Sendungsbewusstsein in Anspruch, d. h. es platziert sich als eine Botschaft. Es handelt sich um eine strategisch gesehen äußerst geschickte Platzierung, um von einem möglichst großen Publikum wahrgenommen zu werden. Für Reisende stellt es zugleich eine Wegmarkierung dar, also einen einfach zu identifizierenden Ort der Orientierung. Berücksichtigt man hier, dass es sich um ein Schulhaus handelt, verkörpert das Gebäude unweigerlich auch eine Repräsentation der Staatlichkeit. Dies alles erreicht das Haus nicht nur durch die prominente Platzierung im Innenwinkel der Weggabelung. Verstärkt wird dies durch die Rückversetzung von den beiden Straßen.

Die massive Bauart in Sandstein verweist darauf, dass das Gebäude etwa zeitgleich mit den Arbeiterhäusern im Norden und zeitlich vor den verputzten Mietwohnungen der Umgebung gebaut wurde. In dieser Relation ist auch die Größe des Gebäudes zu sehen. Im Vergleich zu der Reihe von kleinen Einfamilienhäusern wirkt das Gebäude geradezu kolossal.

Der Perimeter ist ein Gebiet, das sich im Gegensatz zur relativ einheitlichen Bebauung der Umgebung durch seine Größe und seine funktionale Differenziertheit auszeichnet. Der Perimeter unterscheidet sich nicht nur in der Form gegenüber der eintönigen Wohnumgebung. Er ist auch in seinen unterschiedli- 
chen Funktionsflächen ausdifferenziert. Zugleich liegt das Gebiet an der Schnittstelle dreier Nachbarschaftsgebiete, die in sich räumlich jeweils unterschiedlich strukturiert sind. Dies wird aus der Ausrichtung, Bauart und den Gebäudegrößen ersichtlich. Die unterschiedlichen Quartierteile bestehen aus Häusern, die für unterschiedliche Wohnformen konzipiert worden sind. Sie spiegeln damit nicht nur schicht- und milieuspezifische Unterschiede, sondern auch historischsozioökonomische Entwicklungen. Das lässt sich an den kleinen Arbeiter- und Gewerbehäusern ablesen, deren Gärten auf eine ergänzende Subsistenzwirtschaft hinweisen, im Kontrast zu den neueren, reinen Wohnhäusern. Es kann gemutmaßt werden, dass sie unabhängig von aktuellen Gentrifizierungsprozessen von unterschiedlichen sozialen Gruppen bewohnt wurden und werden und dass sie einen eigenständigen Quartierscharakter als räumlich fundierte Identitäten einer Nachbarschaft haben. Damit nimmt die Schulanlage für diese drei Gebiete einen Doppelcharakter als Trennendes und Verbindendes zugleich ein. Es trennt sie in jeweils in sich abgegrenzte Nachbarschaften. Und es stellt zugleich das einzige Gebiet dar, in der ein Aufenthalt in Muße außerhalb des Wohnraums möglich ist. Damit ist es ein Orientierungspunkt für alle außerhäusigen Praktiken, ein Raum, in dem geplante und ungeplante Interaktionen stattfinden können. Es kann einen Treffpunkt oder ein kleinräumiges Zentrum für das öffentliche Leben der drei differenten Quartiersformationen bilden.

Kann die Struktur der Nachbarschaften, die der Architektur eingeschrieben scheint, unter den Bedingungen heutiger mobiler und fragmentierter Lebenszusammenhänge noch aufrechterhalten werden? Die Kongruenzannahme zwischen Flächen- und Sozialraum ist laut Hüllemann et al. (2015: 31) eine nicht haltbare Homogenitätsannahme. Dies leitet er daraus ab, dass sich die Lebensräume nur noch in Ausnahmen auf kleinräumige Stadtteile beschränken. Berufliche Mobilität, Freizeitverhalten, Hobbys usw. fragmentieren die Lebensräume. Dem hält Siebel entgegen, dass die Nachbarschaften in ihrer Relevanz nicht verschwinden, sie nehmen aber andere Formen an: „Früher war Nachbarschaft Schicksal, heute ist sie wählbar. Früher war Nachbarschaft eine räumliche Tatsache, die sich sozial organisiert. Heute ist sie eine soziale Tatsache, die sich räumlich organisiert“" (Siebel 2009: 17). In dieser Perspektive stellt sich ein doppelter Bezug zur Einrichtung Schule her. Da in der Schweiz keine freie Schulwahl besteht, wird bei der Entscheidung über den Wohnort die Schule im Quartier als Auswahlkriterium miteinbezogen, zumindest für diejenigen, die es sich leisten können. Die 
Schulwahl findet umgekehrt - nicht ausschließlich, aber auch - über die Wahl des Wohnortes statt. Regionale Bildungsdisparitäten schreiben sich damit auch kleinräumig in den Raum ein. Das zweite Merkmal ist darin zu sehen, dass die Nachbarschaften für Erwachsene und Kinder beziehungsweise für Familien einen unterschiedlichen Stellenwert haben und daher differenzierter zu betrachten sind. Heutzutage setzt sich für Kinder die Peer Group nicht mehr ausschließlich und zwingend aus der Nachbarschaft zusammen (vgl. Kogler 2015). Die Schulklasse konstituiert jedoch nach wie vor die sozialisatorisch bedeutende Peer Group, und für diese sind die Nachbarschaften der Ort, aus dem sie sich rekrutiert. Auch wenn die Nachbarschaften nicht mehr unveränderliches Schicksal sind, so sind sie deswegen nicht als obsolet zu betrachten. Eher das Gegenteil ist der Fall. Indem die Nachbarschaft zu einem Teil der gestaltbaren Lebenswelt wird, gewinnt sie zusätzlich an Bedeutung. Die Schule spielt dabei in zweifacher Hinsicht eine Rolle: Sie ist für Familien Teil der Wohnortwahl und sie gibt dem Stadtteil ein identitätsstiftendes Gesicht.

Das zeigt sich im Fall Breitenrain besonders deutlich: Die Schulanlage ist die einzige öffentliche Grünfläche auf dem Luftbildausschnitt. Der Park und damit auch die angrenzenden Gebäudekomplexe sind ein Residuum des öffentlichen Raums, das nicht überbaut oder dem Verkehr überlassen ist. Der Park und Teile der Schulanlage bilden damit einen Kontrapunkt zur Umgebung. Es ist der einzige Ort, an dem ein zweckfreier Aufenthalt im Freien vorgesehen ist, an dem Treffen und Interaktionen in der Gemeinschaft im Außenraum stattfinden können. Parks und Spielplätze stellen geradezu prototypisch Räume für gemeinschaftliche Tätigkeiten dar. Sie sind Treffpunkte zwischen Freunden und Familien, aber auch zwischen Fremden, ohne dass sich diese in rollenförmigen Interaktionskonstellationen begegnen. Damit wird die Schulanlage ein wichtiger Bezugspunkt für die Umgebung. Dies kann man sich gedankenexperimentell einfach daran klarmachen, wenn man die Schulanlage wegdenkt. Damit verbleiben - abgesehen von der Kirche und den Straßen - keine öffentlichen Räume im Kartenausschnitt.

In der Betrachtung der mittleren Reichweite des Gebäudekomplexes und der zugehörigen Grünanlage ist das betrachtete Areal als einzige unbebaute beziehungsweise nur teilweise bebaute Fläche auszumachen. Sie verkörpert die übrig gebliebenen Reste eines unbebauten Raums. Es ist der einzige Ort, abgesehen von den Verkehrswegen, wo man sich draußen aufhalten kann. In diesem 
Sinne ist es ein Raum, in dem die Möglichkeiten von zweckfreiem Aufenthalt und gemeinschaftlichen Aktivitäten gegeben sind. Die Verortung des Parks und der Schule an einem zentralen Knotenpunkt konstituiert ein verbindendes Glied einer größeren Gemeinschaft, die durch Straßenzüge in kleinräumige Nachbarschaften ein- und abgrenzt sind. Deren Grenzen sind in der Regel identisch mit den Sichtweiten. Durch ihre Positionalität verbindet die Schule die drei angrenzenden Quartiere und schafft damit einen sozialen Raum im Sinne eines gemeinsamen Anziehungspunkts, der über die sichtbare Innen-Außen-Abgrenzung hinauswirkt. Umgekehrt betrachtet verfügt die Schulanlage und das dazugehörige Areal über eine Sendung oder eine Sogwirkung, die über die reine Sichtbarkeit hinausreicht.

Die Schulanlage lässt sich nicht nur ausschließlich über die Funktionalität innerhalb des Siedlungsgefüges als bedeutsamen Ort rekonstruieren. Da das Gebiet rund um die Schulanlage wie ein markantes Momentum inmitten gleichförmiger Wohnbauten wirkt, kann man davon ausgehen, dass die Schule auch für die Stadtteilidentität zentral ist. Die Schule stellt eine Markierung in der Stadtlandschaft, dem Quartier dar, das diesem damit auch ein Gesicht jenseits der Eintönigkeit der Wohnblöcke gibt.

Aus dem relativ einheitlichen Erscheinungsbild des Stadtteils, was Bauvolumen und Stil der Häuser betrifft, kann gefolgert werden, dass die Häuser zur selben Zeit erbaut wurden und dass in dem Gebiet eine Gesamtplanung stattfand. Dies ist insofern von Bedeutung, als aus der Lage der Schule, der Form des Schulgeländes, aber auch aus dem neoklassizistischen Baustil des Schulgebäudes rekonstruiert werden kann, dass die Schule älteren Datums ist. Damit hat das Schulhaus den Charakter eines Pionierbaus. Die Positionalität der Schule muss als städtebaulich geplant und bewusst gesetzt betrachtet werden. Für die Planung und Errichtung eines solchen Gebäudetyps bedeutet dies, dass ein Perimeter vor der Überbauung ausgegrenzt worden sein muss oder ein Teil nachträglich dazugeschlagen wurde, was die ungewöhnliche Form erklären könnte. Aus dem hohen Raumbedarf kann geschlossen werden, dass es sich bei Schulbauten generell um erschließende Bauten handelt, da der enorme Platzbedarf nachträglich nur in Ausnahmen zurückgewonnen werden kann. Es sind Bauten, die aufs freie Feld gebaut werden. Dabei ist dieser Umstand dialektisch zu sehen. Bei den meisten Schulbauten - zumindest in der Stadt Bern - war dies jeweils der Fall, obwohl die Anzahl der Schulbauten dem Bevölkerungswachstum und den wachsenden 
Schülerzahlen ab Mitte des 18. Jahrhunderts stetig hinterherhinkte und die bestehenden Schulbauten notorisch überfüllt waren (Tögel 2004: 257). Dies gilt auch für die aktuelle Dekade, in der der Stadt Bern vorgeworfen wird, die Schulraumplanung versäumt zu haben. ${ }^{27}$ Die Schulhäuser wurden (und werden teilweise immer noch) jeweils an den äußeren Grenzen der Siedlungen errichtet, wo sie dann relativ rasch von neu gebauten Häusern eingemittet wurden (vgl. die Fallrekonstruktion Hinter Gärten). Damit werden Schulbauten intendiert oder unbeabsichtigt zu ,Pionierbauten“ bei der Besiedlung von Raum. Sie sind als Infrastrukturbauten Teil der Erschließung und Urbanisierung neuer Gebiete. Das bedeutet, dass die Position der Schulanlage einer städtebaulichen Planung entspringt und damit Teil einer Stadt- und Bevölkerungspolitik ist. Das gilt sowohl für den Fall, dass das Schulhaus auf die grüne Wiese gebaut wurde - also einer aktiven, intendierten Urbanisierung -, als auch wenn es am Rand einer bereits wachsenden Stadt erstellt wurde. Auch in der zweiten Variante verkörpert die Schule einen Teil staatlicher Bevölkerungspolitik, wenn diese auch eher reaktiv agierend sein mag. So zeigt sich eine Koinzidenz zwischen Schulhausplanung und Bildungspolitik auf der einen sowie Städteplanung und Bevölkerungspolitik auf der anderen Seite. Schulbauten werden meist in Phasen der Bildungsexpansionen gebaut, die mit Phasen demographischen Wachstums oder mit Phasen ökonomischer Strukturtransformationen einhergehen. Die prominente Platzierung des Schulhauses, die primär symbolischen Charakter hat und es damit sichtbar platziert, kann als Geflecht zwischen Bildungs-, Bevölkerungs- und Biopolitik interpretiert werden. Das Schulhaus stellt einen sichtbaren Markstein einer zukünftigen und geplanten Gesellschaft dar: Es ist ein Bau, der in die Zukunft gerichtet ist beziehungsweise der auch in den gestalterischen Mitteln die Zukunft darstellen soll. Dieser Gesichtspunkt soll in die Analyse der Architektur der Gebäude mitgenommen werden. Es gilt die Schulbauten dahingehend zu befragen, welche Sinnstrukturen als zukünftige Anforderungen und Erfordernisse von sozialisierten Subjekten aus der Architektur rekonstruiert werden können.

Aus der Positionierung und der Umgebung der Schule wurde rekonstruiert, dass das Schulhaus in der räumlichen Struktur des urbanen Gebiets einen wesentlichen Bezugspunkt darstellt. Das Schulhaus Breitenrain ist ein markanter 
Ort in der Struktur des Stadtteils und gibt ihm ein Gesicht, indem das Gebäude prominent positioniert ist und zur Monotonie der Umgebung einen Kontrapunkt darstellt. Die Schulanlage oder Teile davon machen den öffentlichen Raum des Stadtteils aus. Die Schule gibt dem Stadtteil ein identitätsstiftendes Gesicht, und sie ist der Ort, an dem gemeinschaftliche Tätigkeiten stattfinden können. Dies wird noch plastischer, wenn man die Schulanlage gedankenexperimentell aus dem Bild löscht. Umgekehrt wird die räumlich-manifest rekonstruierte Verbindung durch die Schule in einem latenten Sinne institutionalisiert. Eine Nachbarschaft als sozialräumlich konstituierte bildet sich erst dadurch, dass Schulen für Familien und Kinder einen wesentlichen und institutionalisierten Bezugspunkt eines Quartiers darstellen. Die Schule als , unsere' Schule ist Teil , unseres ' Stadtteils und Teil des ,Rufs' eines Stadtteils, egal ob im Guten oder Schlechten. Delitz (2010) weist aus einer architektursoziologisch-makrotheoretischen Perspektive darauf hin, dass Architektur der Gesellschaft einen Spiegel vorhält, sie erlaubt der Gesellschaft, sich zu erkennen. Es ist in einem nächsten Schritt also eine Detailbefragung des Gegenstandes vorzunehmen, um Antworten zu bekommen, welches Gesicht mit dem Schulhaus Breitenrain repräsentiert wird.

Um Details herauszuarbeiten, wird im Folgenden auf den vergrößerten Ausschnitt (Abb. 5) fokussiert. Im Fokus der Analyse stehen die Gebäude- und Geländeeinheiten und deren Ausrichtung beziehungsweise gegenseitige Bezugnahme sowie die Frage der Funktionalität der Gebäudeanlage.

\subsection{Schulhausareal Breitenrain - Antagonismus von Unterricht und Nichtunterricht}

Das auffallendste Element des Ausschnitts ist die blaue Farbe des Platzes. Durch die blaue Markierung wird der Platz in zwei annähernd gleich große Hälften unterteilt: Nordseitig ist der Platz blau und südseitig grau. Auf dem Gelände gibt es fünf Bauten, die aus unterschiedlichen Stilepochen zu stammen scheinen und vermutlich nicht zur selben Zeit entstanden sind. Die Gebäude werden in der Reihenfolge ihrer Größe betrachtet. 
Das größte Gebäude, das Schulhaus ${ }^{28}$, ist in massiver Bauweise im neoklassizistischen Stil erbaut. Im Bild sind die repräsentativen Fassaden zu erkennen. Der sichtbare Eingang befindet sich auf der westlichen Stirnseite. Das Gebäude ist längsrichtig streng achsensymmetrisch aufgebaut. Ein Ausreißer aus der Symmetrie ist das Zwerchhaus über dem Eingang. Breitseitig ist die Symmetrie durch die ungleiche Anzahl an Gauben gebrochen. Im Westen, gegen die Straße hin, ist das Haus zurückversetzt, ohne dass davor ein repräsentativer Platz entsteht, wie dies dem Baustil, den Proportionen und der Positionierung nach erwartet werden könnte. Das Gebäude zieht sich an der Breitenrainstraße entlang, von welcher es zurückgesetzt ist.

Nördlich des Schulgebäudes, leicht nach Osten versetzt und in der Ausrichtung rechtwinklig zum Hauptgebäude, befindet sich ein im Riegbau erstelltes Gebäude, das niedriger als das Hochgebäude ist. Die Fenster an der Giebelseite lassen erahnen, dass es sich um ein einstöckiges Gebäude mit einem hohen Raum handelt. Fast mittig angedockt befindet sich ein flacher, mehrteiliger und vieleckiger Annex. Dieser Teil ist dem Gebäude zugehörig beziehungsweise stellt eine später hinzugekommene Funktionserweiterung dar. Der Annex ist mitten in den ehemaligen Hof platziert.

Westlich des Hauptgebäudes steht ein niedriger, schmaler und lang gezogener Bau, der aufgrund des Bauvolumens nur ein Unterstand sein kann.

Bereits erwähnt wurde die Farbgebung, die den Platz in zwei Hälften unterteilt, wobei der größte Teil der unbebauten Fläche blau ist. Die Farbgebung übernimmt eine Funktionszuweisung. Die Bodenmarkierungen lassen erkennen, dass es sich um einen Sportplatz handelt. Der nicht blaue Teil besteht aus unterschiedlichen Fragmenten: eine Art von Umrandung um das Hauptgebäude herum, die Erschließung des Geländes von Süden her und ein vieleckiger Restraum hinter dem Garderobentrakt.

28 Zum besseren Nachvollzug und aus Gründen der abkürzenden Darstellung werden die Gebäude hier in ihren Funktionen benannt. Methodisch-analytisch ist noch zu rekonstruieren, was die Strukturprinzipien sind, welche das Schulhaus als Schulhaus erkennbar machen. 


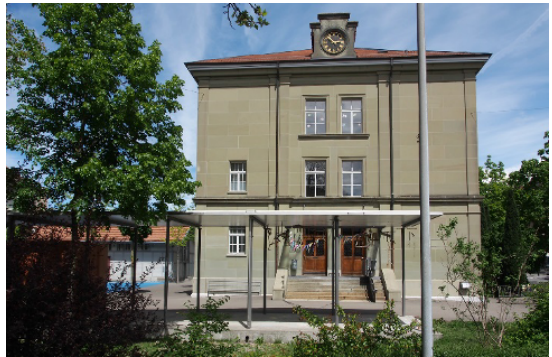

Abb. 6: Schulhaus Breitenrain. Ansicht Südost ${ }^{29}$

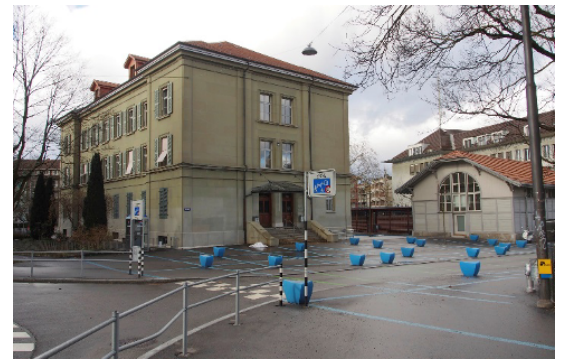

Abb. 7: Schulhaus Breitenrain. Ansicht West

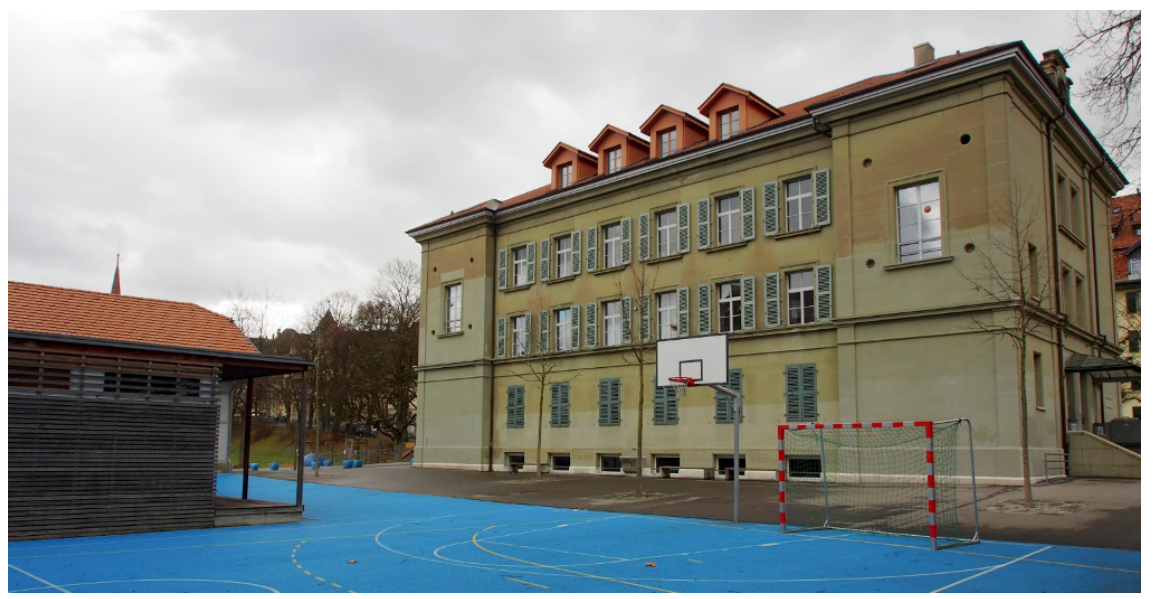

Abb. 8: Schulhaus Breitenrain. Ansicht Nord

29 Wenn nicht explizit anders ausgewiesen, liegt das Copyright der Bilder beim Autor. 


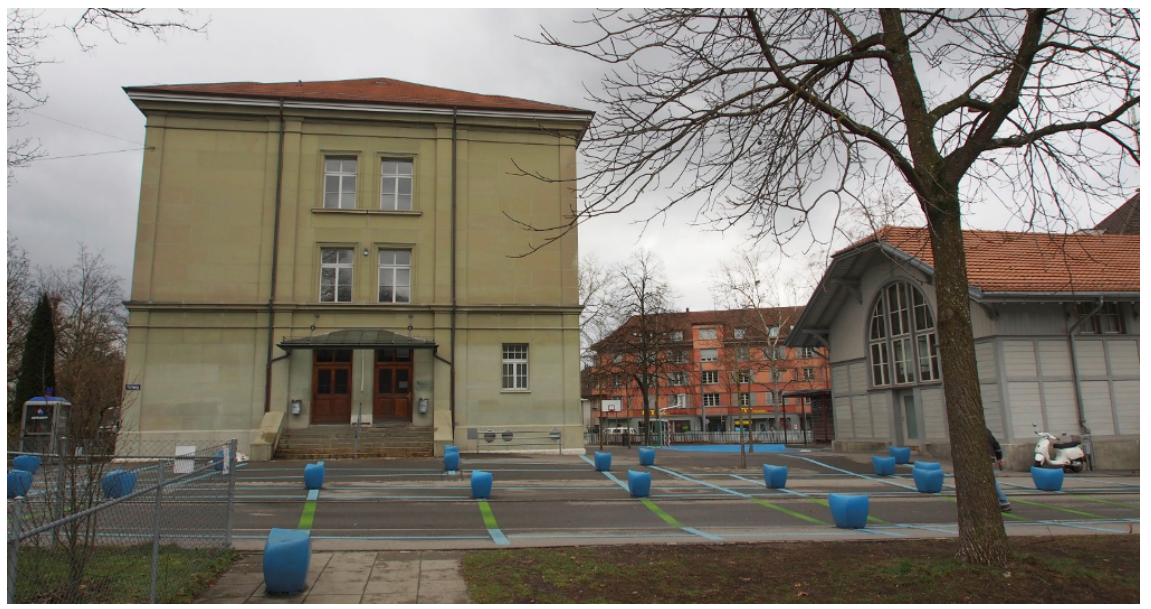

Abb. 9: Schulhaus Breitenrain. Ansicht Ost

Der Schulhausplatz ist auf drei Seiten durch eine Einfriedung abgegrenzt. Der Unterstand auf der Westseite steht wie ein Riegel vor dem Hauptgebäude. Auf der Südseite befindet sich ein Zugang auf das Gelände. Der Zugang verläuft zwischen einem Gebäude, das durch den Zaun von dem Schulgelände abgetrennt ist und einer bepflanzten Abgrenzung. Eine Rabatte mit Bepflanzungen und vier Bäumen befindet sich zwischen Schulhaus und Straße. Sie wird zusätzlich durch einen Zaun abgegrenzt.

Die blaue Farbe des Sportplatzes findet sich auf der östlichen Seite in anderer Form wieder. Der Platz hinter dem Haus, die Gehwege beidseits der Straße und die Straße selbst sind mit einem Geflecht von blauen Linien bedeckt. An den Schnittpunkten sind unregelmäßig blaue Objekte verteilt. Auf der Straße befinden sich eine Fahrbahnschwelle und Markierungen mit Geschwindigkeitsbegrenzungen.

Der Park ist in verschiedene Bereiche gegliedert. Der vordere Bereich ist in vier unterschiedliche Gebiete unterteilt: Ein Bereich im südlichen Teil, in dem rote Beläge und Spielgeräte sichtbar sind; ein Teil im nördlichen Abschnitt, der einen abgedeckten Sandkasten enthält, und dazwischen eine größere Rasenfläche, deren nördlicher Teil ein hügeliges Gelände darstellt. Als Fortsetzung des Weges aus dem Gebäude ist ein geradliniger Pfad ersichtlich, der zu einem Tor im Zaun führt, der den hinteren Teil der Rasenfläche abtrennt. Hier sind auch 
Fußballtore erkennbar. Vom Schulhof aus führt der Weg entweder zum Sandkasten oder zu den Spielgeräten. Zwei gekrümmte Weg verlaufen von Nord nach Süd und enden scheinbar unmotiviert.

Zum besseren Nachvollzug und aus Gründen der abkürzenden Darstellung wurden die Gebäude bisher in ihren Funktionen benannt, auch wenn nicht rekonstruiert wurde, wieso diese Gebäudegruppe eine Schulanlage darstellt. Für die Untersuchungsanlage und für die Methode der objektiv hermeneutischen Sequenzanalyse ist es wesentlich, die Strukturprinzipien zu rekonstruieren, was die Schule als Schule erkennbar macht. ${ }^{30}$

Vor der Analyse ist die Sequenzialität festzulegen. Eine Möglichkeit ist es, dem ikonographischen Pfad zu folgen und die Elemente zu fokussieren, die am auffälligsten sind. Ins Auge springen beim Haupthaus vor allem die Massivität und der Farbkontrast des Dachs. Als weitere auffällige Elemente sind der blaue Sportplatz und die Turnhalle zu nennen. Um die Analyse abkürzend darzustellen, wird teilweise illustrierend zusätzliches Material verwendet. Weitere Möglichkeiten der Sequenzierung wäre, der Größe der Gebäude zu folgen oder einen Pfad festzulegen, etwa wie man sich dem Gebäude idealtypisch annähert. Die Sequenzialität kann dem Arrangement von mehreren, zu unterschiedlichen Zeiten entstandenen, hinzugekommenen Gebäudeteilen folgen, welche gewissermaBen eine naturwüchsige Diachronie darstellen. Die Architektur wird dabei als Baukette über mehrere Zeitpunkte hinweg analysiert. Das erlaubt einen räumlichen Prozess so zu begreifen, dass die Gebäude und ihre Umgebung in ihrer Verflechtung mit den Entwicklungen der Schule und mit den gesellschaftlichen Entwicklungen betrachtet werden. Zudem gibt es vor allem auch Auskunft über die sukzessiv hinzugekommenen oder Neudefinitionen von schulischen Funktionen, die sich in Raum(an)ordnungen von Gebäuden zeigen.

Die Sequenzialität wird so ausgelegt, dass sie in groben Zügen von außen nach innen angelegt ist. Daher wird mit den Anordnungen der Gebäude und Elemente innerhalb der Schulanlage begonnen. Danach wird der Fokus auf die

30 Im methodischen Vorgehen ist es nicht erlaubt, Kontextwissen herbeizuziehen. Dass es sich um ein Schulhaus handelt, ist, in diesem Fall kein Kontextwissen. Das Haus ist im Gegensatz zu anderen Fällen unverkennbar als Schulhaus auszumachen. Nichtsdestotrotz oder gerade darum sollen die Strukturprinzipien rekonstruiert werden, warum das Haus intuitiv und unverzüglich als Schulhaus erkannt wird. 
Repräsentation von Schule gelegt, also dem, was nach außen repräsentiert wird. Zum Schluss folgt eine Analyse der Raumordnungen und der Binnenfunktion des Schulgebäudes.

\subsubsection{Funktionserweiterungen der Schule}

Bevor auf die einzelnen Teile eingegangen wird, ist zu fragen, wieso das Gebiet überhaupt ein Ganzes darstellt. Erst wenn dies geklärt ist, kann die Frage der (An-)Ordnung angegangen werden, in welcher die symbolischen und funktionalen Bezüge innerhalb der Gebäudegruppe vorangenommen werden. Wieso stellen diese Gebäude eine Einheit und eine Schule dar? Ein erster Grund ist in der Form des Perimeters zu sehen. Für eine Einheit spricht, dass das Gebiet auf einem in sich abgegrenzten Grundstück steht, das auf drei Seiten durch Straßen und im Norden durch ein Gebäude abgetrennt wird. Dabei ist festzustellen, dass der Perimeter in zwei annähernd gleich große Teile geschnitten ist, was durch die farbliche Hervorhebung in einem grellen Blauton unmissverständlich und allgegenwärtig unterstrichen wird. Diese Zweiteilung, so kann konstatiert werden, muss für die Schule ein zentrales Strukturmerkmal darstellen.

Das zweite Argument findet sich in der außeralltäglichen und außergewöhnlichen Farbe des Schulplatzes. Es ist das Sportfeld und nicht etwa das Schulhaus, welches farblich hervorgehoben wird. Damit wird ihm explizit eine herausragende Bedeutung zugeschrieben. Die farbliche Markierung wird an anderer Stelle wiederaufgenommen. Hinter dem Haus, also auf der östlichen Seite, führen Linien und blaue Objekte auf das gegenüberliegende Gelände, wo sich ein weiteres rundes Objekt in derselben Farbe befindet. Damit wird optisch eine Gemeinsamkeit farblich markiert.

Wenn die Gebäude genauer betrachtet werden, ist festzustellen, dass sie sich in fast allen sicht- und denkbaren Kategorien unterscheiden: Alter, Form, Größe, Volumen, Farbe, Baustoffe, Baustil, Ausrichtung, Anzahl der Fenster und Funktionen, soweit dies aus den Gebäudehüllen erschlossen werden kann. Heterogenität zeigt sich auch bei den unbebauten Flächen. Auch hier finden sich verschiedene in sich abgegrenzte und klar umrissene Felder, die sich klar in Form, Farbe, Materialität, Bepflanzung unterscheiden und die unterschiedliche Funktionsflächen darstellen. Gemeinsam ist den Teilen, dass sie als gestaltete, funktional 
eindeutige Bereiche erkannt werden können. Sie scheinen entweder für spezifische Praktiken geplant und gebaut, die zum Beispiel das Schaukeln beinhalten. Oder sie dienen als Markierung von Grenzen, wie dies bei dem Staudenbeet auf der Südseite des Hauptgebäudes sowie bei einer etwas verborgenen Rasenfläche zwischen Straße und Unterstand der Fall ist. Insgesamt ist eine große Vielfalt von Volumen, Formen und Flächen zu konstatieren. Was das Gesamte als Einheit ausmacht, ist also die vielseitige Zusammensetzung unterschiedlicher Bauten und Flächen. Die Konstitution als Gesamtheit ergibt sich gerade aus dieser Zusammensetzung verschiedener spezifischer Funktionen in Form von Bauten und Flächen, die jeweils einen intentionalen Gehalt besitzen, und Elementen, die eine Innen-Außen-Abgrenzung unterschiedlicher Durchlässigkeit darstellen.

\subsubsection{Inklusion und Exklusion durch Positionalität und Raumordnungen}

Es ist festzuhalten, dass die Gebäude in Größe, Monumentalität der Bauart und in der Positionierung eine hierarchische Rangordnung beinhalten. Das Hauptgebäude steht massig und mächtig direkt der Öffentlichkeit zugewandt an der Straße. Damit wird diese Seite zur öffentlichen Seite des Gebäudes, zum der Öffentlichkeit zugewandten ,Gesicht‘. Das größte ist auch als das älteste Gebäude erkennbar. Es ist unverkennbar der zentrale Sitz der Schule. Insgesamt ist die Lage des Hauses mit derjenigen einer herrschaftlichen Villa vergleichbar, die ihre Repräsentativität nach außen sichtbar darstellt. Es liegt leicht zurückversetzt von der Straße, was eine gewisse Privatheit herstellt und bereits den Zugang mit einer Exklusivität belegt. Zwischen öffentlicher Straße und der Privatheit des Gebäudes entsteht eine halb öffentliche Zone, eine Art Puffer, die man auf dem Weg zum Gebäude betreten muss. Wer sich dem Gebäude nähern will, tut sich vor dem Erreichen des Gebäudes kund. Zugleich wird die Repräsentativität des Gebäudes durch die vorgelagerte Bepflanzung verstärkt: Das Gebäude ist distinktiv zurückversetzt und kann es sich ,leisten', sich mit Bepflanzungen zu schmücken. Umgekehrt stellt eine Bepflanzung auch ein repräsentatives Aushängeschild gegenüber der Öffentlichkeit dar. Die Positionierung richtet sich an die Öffentlichkeit. Zugleich konstituiert die Position an der öffentlichkeitsabgewandten Seite eine - ehemals - große (Frei-)Fläche. Diese Fläche gehört zur inneren Funktionalität des Geländes; sie ist auf der privaten Seite des Gebäudes situiert. 
Man kann sich gedankenexperimentell überlegen, welche Bedeutungsgehalte andere Positionen des Hauptgebäudes vermitteln.

Sowohl eine Positionierung in der Mitte des Perimeters als auch im hinteren Teil des Geländes hätten eine viel stärkere Abgrenzung in die Privatheit und damit einen stärkeren Selbstbezug des Hauses zur Folge. Für das Schulhaus Breitenrain ist also wesentlich, dass es sich in Position, Ausrichtung und Baustil an die Öffentlichkeit gewandt positioniert. Es ist ein Gebäude, das sich geradezu an die Öffentlichkeit richtet. Die Positionierung, die einen großen abgegrenzten Binnenraum schafft, verweist zugleich darauf, dass sich das Gebäude in seiner Funktionalität abschließt. Es hat einen Binnenraum, der sich von der Öffentlichkeit absetzt.

Diese Struktur tritt noch konturierter hervor, wenn man die Erschließungen des Gebäudes betrachtet. Im Vergleich zu allen anderen (Wohn-)Gebäuden des Quartiers sind die Erschließungen nicht direkt von der Straße her zugänglich. Sie sind jeweils an den Stirnseiten des Gebäudes angebracht. Das Schulhaus öffnet sich nach vorne und hinten. Die Ausrichtung des Gebäudes und die ErschlieBungswege sind nicht identisch. Im Gegenteil wird der direkte Weg zum Eingang mehrfach verstellt: optisch und physisch durch das Kioskhäuschen an der Kreuzung, durch einen Zaun und durch den dahinterliegenden Fahrradunterstand.

Der Zugang eröffnet sich erst, wenn man seitwärts vor ihm steht. Das Gebäude positioniert sich für Fremde somit wenig einladend. Zwar verfügt es über einladend gestaltete Zugänge, die so angelegt sind, dass sie Vorbeikommende zum Eintreten bitten. Die Zugänge sind so gestaltet, dass sie nur von denjenigen benutzt werden, die die Wege kennen. Das Gebäude schottet sich gegenüber fremden Besucher_innen ab.

Das Schulareal wurde bis hierhin als abgegrenzter Bereich rekonstruiert. Adressiert wird eine spezifische Gruppe: die Schüler_innen. Gäste sind nicht vorgesehen. Zudem wurde festgehalten, dass die Schulanlage aus zahlreichen einzelnen Teilen besteht, die sukzessive hinzugekommen sind. Schulen können damit als Antwort auf ein vielschichtiges Handlungsproblem verstanden werden, in welchem sich unterschiedlichste Bereiche ausdifferenziert haben. Dabei ist bezeichnend, dass die verschiedenen Bereiche nach einer relativ großen Anzahl architektonischer Artefakte verlangen, die differente Handlungsintentionen enthalten. Aus der Vielfalt und Anzahl der Felder kann geschlossen werden, dass die einzelnen Bereiche relativ eindeutige und damit klar umrissene Möglichkeits- 
räume darstellen. Ein Klettergerüst ist eine architektonische Geste, die primär zum Klettern einlädt, und das Sportfeld ist eine Geste, die zum Ballspiel einlädt. Es sind Bereiche, die durch Architektur zu bestimmten eindeutigen Tätigkeiten animieren. Die Schulanlage stellt insgesamt eine Anlage dar, die sich durch Spezialisierungen auszeichnet. Die Einheit ist eine Zusammensetzung unterschiedlichster Teile, die jeweils für sich relativ klar umrissen und abgegrenzt sind.

Im Weiteren kann geschlossen werden, dass die Schule für eine große Menschenansammlung erbaut ist. Die Schule ist eine Organisation, die eine zentralistische Tendenz hat. An einem Ort wird eine relativ große Anzahl von Schüler_innen gebündelt. Als Erkenntnis scheint dies ziemlich banal. Es ist insofern nicht banal, als im Prinzip auch andere Modelle der Unterrichtung denkbar wären. Zudem prägt die Wahl großer Schulhäuser und zentraler Schulen die pädagogischen Möglichkeiten ganz entscheidend. Historisch gesehen war über Hunderte von Jahren das Hauslehrermodell das dominante pädagogische Konzept. Die Diskussion fand auch zu Zeiten statt, wo das staatliche Schulsystem entwickelt wurde. Noch Herbart favorisierte ein Schulmodell, welches nicht als zentralistisch organisiertes Schulmonopol aufgebaut ist, sondern ein Modell, in dem die Schule von den Eltern in nachbarschaftlichen Initiativen organisiert werden sollte (siehe Busse \& Helsper 2007: 324). Für die Genese und Entwicklung der Schulen in ihren heutigen Formen ist der zentralistische Gedanke des Aufbaus von Schulen äußerst prägend. Er ist für die räumliche Manifestation der Schule in ihren spezifischen Architekturen genauso konstitutiv wie für die Ausrichtung der Schule und des Bildungssystems als uniforme und normierende Massenveranstaltung.

Doch kehren wir zurück zur Frage der ausdifferenzierten Funktionsbereiche. Die Größe der Schule und die unterschiedlichen Funktionsbereiche lassen auch darauf schließen, dass die Schule eine komplexe arbeitsteilige Organisation darstellt. Das räumliche Organisationsprinzip mit funktional differenten, jeweils eindeutigen Bereichen lässt vermuten, dass die Schule auch funktional differentes, jeweils spezialisiertes Personal braucht. Aus den Luftaufnahmen kann also erschlossen werden, dass die Schule sowohl für das manifeste städtebauliche Bild, als auch für die latente gesellschaftliche Organisation wesentlich ist. Dabei ist das Schulhaus der Sitz der Organisation. Bei der Betrachtung der unterschiedlichen Teile wird daher mit dem Sitz begonnen, um zu rekonstruieren, welche Bedeutungsgehalte das Gebäude repräsentiert. 


\subsection{Schulgebäude Breitenrain - Sinnbild einer Schulkaserne}

Das Hauptgebäude ist im neoklassizistischen Stil erbaut (Abb. 6-10). Es hat eine klare Bauweise, die auf Symmetrie angelegt ist. Über einem rechteckigen Grundriss erhebt sich das Gebäude drei Geschosse hoch. An den beiden Enden des Grundrisses sind zwei quergestellte Teile angebracht, die von der Breitseite betrachtet als Risalite erkennbar sind.

Das Gebäude ist gefertigt aus großen gefugten Sandsteinquadern. Horizontal werden die Wandflächen in drei Bereiche getrennt: Ein hüfthoher Sockel aus Kalkstein bildet die Basis. Auf diesem befindet sich das als Hochparterre angelegte Erdgeschoss, welches durch ein Sohlbankgesims von den oberen beiden Geschossen abgetrennt ist. Der Wandabschluss wird durch ein Dachgesims und ein etwas stärker ausgebildetes Traufgesims gebildet, das von einem leicht vorkragenden Walmdach abgeschlossen wird.

Vertikal wird das Gebäude durch die symmetrisch angeordneten Eckrisalite gegliedert. Diese wirken gestaltgebend. Sie bilden turmgleiche Pfeiler und rahmen sowohl die Eingangsbereiche an den Giebelseiten wie die Fensterfronten an den Längsseiten.

In den beiden quergestellten Bauteilen sind die horizontale und vertikale Erschließung untergebracht: zwei identische Doppelportale und die Treppenhäuser. Eine Treppe führt zu den Türen hoch. Zusätzlich ist über den Türen ein kleines Vordach angebracht. Die Türen sind in massivem Eichenholz gefertigt und haben vier eingelassene Fenster. Über den Türen befinden sich in jedem Stockwerk zwei Fenster. Die Fenster im zweiten Stock werden durch eine gemeinsame Sohlbank zusammengehalten. Zudem wird die horizontale Dreiteilung der Fassade durch die beiden Eckrisalite und durch die Fallrohre zusätzlich unterstrichen. Asymmetrisch angebracht, hat es ein beziehungsweise zwei Fenster an jeweils einem der Eckrisalite. Der markanteste Unterschied an den beiden Stirnseiten ist das Zwerchhaus über dem Eingang an der Westseite des Gebäudes, das eine Uhr trägt.

Auch die Breitseiten zeichnen sich durch Symmetrie aus. Auf jedem Stockwerk befinden sich im von den Risaliten flankierten Mittelbau sechs Fens- 
ter, mit jeweils identischem Abstand, was sechs Achsen durch das Gebäude hindurchzieht. ${ }^{31}$ Dem entspricht die Anordnung der Kellerfenster. Unterschiedliche Achsen sind auf dem Dach festzustellen. Auf der Südseite sind zwei Gauben angebracht, auf der Nordseite sind es deren vier.

Die vertikale Erschließung befindet sich in den Risaliten. An den Risaliten sind asymmetrisch Fenster angebracht: auf der Nordseite zwei große Fenster auf der Höhe eines Zwischengeschosses; auf der Südseite je ein Fenster pro Stockwerk. Die horizontalen Erschließungen erfolgen zweispännig. Das heißt, hinter den Eingangsbereichen sind die Zugänge zu je zwei gleich großen Zimmern, die im Mittelteil angebracht sind. Im Erdgeschoss sind drei Schulzimmer eingerichtet. Das südöstlich gelegene Zimmer ist als Esszimmer mit Küchenkombination eingerichtet. In den beiden Obergeschossen sind vier gleichartige Schulzimmer untergebracht. Die Klassenzimmer sind durch eine Verbindungstür verbunden. Die eingezeichnete Frontalbestuhlung zeigt, dass diese Verbindungstür im Rücken der Schüler_innen liegt. Auf der Westseite hat es einen Lift. In den Risaliten befindet sich pro Stockwerk ein kleinerer abgetrennter Raum, der mit Tischen und mehreren Stühlen eingerichtet ist.

Das Gebäude ist in repräsentativer Bauweise errichtet. Es spricht eine spätklassizistische Formensprache, die auf klare, schnörkellose Formen und strenge Symmetrie angelegt ist. Die reine Symmetrie steht aber nicht nur für den klassizistischen Baustil in seinen unterschiedlichen Formen, sie ist auch ein charakteristisches Stilmittel von Herrschaftsbauten. Produktionsgebäude wie Bauernhäuser, Handwerkerhäuser und dergleichen sind in der Regel schon aus ihrer Funktionalität heraus nicht symmetrisch, da sie oft einen Wohn- und einen Ökonomieteil vereinen.

In einer ersten Assoziation wirkt der Bau durch die massive Bauart und die Architektur kasernenartig. Die stirnseitig angebrachten Erschließungen verstärken diesen Eindruck, da sie eine Serie einheitlicher, gleichartiger Räume erwarten lassen. Der Bau assoziiert also keinen Regierungssitz, sondern in diesem Vergleich die Unterbringung von Soldaten beziehungsweise der Armee, welche

31 Auf dem Bild sind die Fensterläden im Hochparterre geschlossen. Dies ist dem Umstand geschuldet, dass ich aus Gründen des Datenschutzes die Bilder von leeren Schulhäusern gemacht habe. Sie sind während der Schulferien entstanden. 
die Schutzmacht oder das Angriffspotenzial des Staates darstellt. Der Bau wirkt nicht nur durch die Strenge der Symmetrie assoziativ wie ein Herrschaftsbau. Es sind vor allem die Bauart und die Materialien, die den Bau kantig, stoisch und beherrschend erscheinen lassen. Das Haus ist für eine Dauer gebaut, die mehrere Generationen über den Lebenshorizont der Erbauer hinausreicht.

Auffallend ist, dass das Gebäude zwei sehr unterschiedliche Gesichter hat, je nachdem, ob die Breit- oder die Stirnseite betrachtet wird. Zudem unterscheiden sich auch die beiden Breitseiten, je nachdem, ob sie von vorne, also straßenseitig, oder von hinten betrachtet werden. Das verbindende Merkmal sind die Eckrisalite, die sowohl von der Längs- auch als von der Breitseite sichtbar sind. Die Risalite ragen lediglich um Mauerbreite hervor und bringen damit keinen Raumgewinn. Sie haben eine rein symbolische Funktion. Das Haus nimmt damit eine Anlehnung an eine stilisierte Burg mit vier Ecktürmen. Dies kommt insbesondere an den Stirnseiten gesteigert zur Geltung, wo die Risalite pfeiler- und turmgleich die Erschließungen rahmen. Indem die Risalite auf den Stirnseiten kleine und unregelmäßig angebrachte Fenster haben, wird der Eindruck einer Trutzburg verstärkt. Das Haus, das sich schon durch seine Lage abkapselt, ist auch in seiner Gestaltung unzugänglich.

Die Eingangsbereiche sind nicht ebenerdig angebracht. Eine Treppe führt zum Eingang hinauf. Die Aufgänge zum Hochparterre, der Portalcharakter der Erschließung, die Doppeltüren und die darüberliegenden Fenster erzeugen einen vertikalen Sog. Ein Mensch, der sich vor dem Gebäude befindet, wirkt damit besonders klein. Es ist ein Gestaltungsprinzip, das monumentale Herrschaftsund Sakralbauten auszeichnet, welche eine Wirkung suggerieren, die ,jeden Besucher bereits beim Eintritt einen Kopf kleiner machen“ (Pelzer, zit. nach Rieger-Ladich \& Ricken 2009: 186). Durch das Besteigen der Treppe muss der Blick gehoben werden. Dem Eintretenden wird eine Leistung abverlangt. Die erhöhte Lage und die Anordnung der Fenster erlauben dem Auenstehenden keinen Einblick, während die kleinen, ausguckgleichen Fenster dem Innenstehenden erlauben, den Ankömmling frühzeitig zu sehen. Die kleinen Fenster an den Stirnseiten sind eines der wenigen asymmetrisch angebrachten Elemente. Sie sind kleiner als die anderen Fenster, haben weder Sturz noch Rahmen und verfügen über eine doppelt so dichte Sprossenaufteilung, was sie aus der Ferne wie vergittert wirken lässt. Sie wirken, als wären sie nicht Teil des symmetrisch gestalteten Arrangements, sondern aus rein funktionalen Gründen erstellt worden, 
um den Blick auf die Ankommenden zu gewährleisten. Insgesamt kann gesagt werden, dass die beiden Längsseiten trotz der durchaus großzügigen ErschlieBungen keine einladende Wirkung haben. Ohne explizite Ein- oder gar Vorladung wird kein zufällig Vorübergehender eintreten.

Ein kleines und zuoberst angebrachtes Element ist die auf der Westseite angebrachte Uhr. Sie ist das bestimmende und herausragende Element dieser Fassade und fungiert als Emblem. Durch das Zwerchhaus wird sie zu einer stilisierten Turmuhr und vereint gleich zwei Embleme von Schulhäusern in sich: Uhr und Turm. Die Uhr verkörpert und repräsentiert die Metrisierung und Rhythmisierung von Zeit. Sie hat eine Doppelfunktion. Funktional als Zeitgeber richtet sie sich an die Bewohner_innen gegenüber und an die vorbeiziehende Öffentlichkeit. Wer auf dem Schulgelände steht, sieht sie nicht. Daher liegt ihre primäre Funktion in der Symbolik, die sie nach außen erzeugt. Wie ein Götzenbild oder wie Gesslers Hut thront sie über dem Eingang. Doch anders als Gesslers Hut, der eine explizite Unterwerfung verlangt, wirkt sie auf die Sozialisanden ungesehen und daher unbewusst sublim in Form der Metrisierung der schulischen Praktiken. Die Uhr verkörpert die äußere, sichtbare Metrisierung, die auf eine innere Metrisierung und Rhythmisierung abzielt. Es objektiviert sich ein Regime, das sich über die zeitliche Struktur Zugriff auf die Subjekte erwirkt. Die Metrisierung der Zeit ist für die Institution Schule ein ganz entscheidendes Disziplinierungsmoment; sie symbolisiert die Metrisierung des Tages, was einer geplanten, rationalen und nach Routinen ausgerichteten Lebensführung entspricht.

Die beiden Breitseiten sind von den gleichen grundlegenden Ordnungsprinzipien der strengen Symmetrie gekennzeichnet. Im Gegensatz zu den Längsseiten dominiert aber der breite Mitteltrakt, der bei den Längsseiten gar nicht sichtbar ist. Die Breitseiten sind der eigentliche innere Kern des Gebäudes. Dieser ist eingerahmt von zwei abweisenden Blöcken. Dabei ist ein wesentlicher Unterschied der beiden Breitseiten zu konstatieren.

Auf der Südseite, die der Straße zugewandt ist, sind überall nach denselben Ordnungsprinzipien Fenster angebracht. Damit wird die Assoziation mit einer Burg wesentlich abgeschwächt. Das Gebäude stellt sich in seiner Funktionalität einheitlich dar, ohne dass Unterschiede der Innenräume von außen erkennbar sind. Das Gebäude suggeriert eine strenge Eintönigkeit sowohl in den Gestaltungsprinzipien als auch in den Raumaufteilungen, wie dies für Kasernen oder 
reine Verwaltungsgebäude typisch ist. Durch die Reihen von Fenstern wirkt das Haus freundlicher, offener und durchlässiger als an den hermetisch abgeriegelten Stirnseiten. Zur Straßenseite hin gibt sich das Haus in seiner Gestaltung als Verwaltungsgebäude. Das Gebäude repräsentiert die staatliche Gewalt. Dies wird einerseits in einer stilisierten Anlehnung an die Machtsymbole feudalistischer Herrschaftsausübung erreicht. Andererseits repräsentiert das Gebäude in Grundriss und Raum(an)ordnung eine rationalisierte Verwaltung. Das Gebäude hat eine Form- und Stilsprache, mit der es durchaus auch etwa als Gerichtsgebäude funktionieren könnte.

Hofseitig ist eine Besonderheit in den Risaliten zu konstatieren (Abb. 8). Anders als auf der Südseite werden die Fensterreihen nicht fortgesetzt. In den Risaliten sind überdimensionierte Fenster angebracht, wodurch sie noch machtvoller wirken. Sie verstärken die Aufteilung des Gebäudes und den turmartigen Charakter. Zudem wirken die unterschiedlichen Maßstäbe, als hausen zweierlei Bewohner_innen in dem Gebäude. Aufgrund der Fenster könnte man folgern, dass im Mitteltrakt ,Zwerge" wohnen, während die flankierenden Risalite für ,Riesen` gebaut sind. Die Proportionen sind gerade umgekehrt zum jeweiligen Platzbedarf. Der große Mitteltrakt kann eine große Zahl von ,Zwergen“ beherbergen. Die Risalite sind ungleich kleiner und haben Platz für höchstens einen ,Riesen“. Das Gebäude stellt nach hinten, also zur Binnenseite, eine Struktur dar, in der viele ,Zwerge" unter der Obhut und Überwachung von je einem ,Riesen“ stehen.

Die Machtasymmetrie, die sich in den Größenverhältnissen findet, reproduziert sich, da sich die Fenster der ,Riesen“ ausschließlich im Obergeschoss befinden. Sie wirken wie ein Ausguck; man hat einen umfassenden Überblick von hier aus. Man kann aber nicht hineinschauen und sieht nicht, ob jemand hinausschaut. In dem Sinne reproduziert sich die Anordnung der Fenster mit derjenigen an den Stirnseiten. Sie haben zumindest teilweise die Wirkung eines Panoptikums (siehe Bentham 2013), eines asymmetrischen Blickregimes, indem die Überwachten einer Struktur gegenüberstehen, die ihrer Überwachung dienen kann, bei der jedoch nicht unmittelbar ersichtlich ist, ob die Überwachung faktisch stattfindet oder nicht. Die Wirkung ist, dass die Überwachung dauerhaft antizipiert und internalisiert wird. Sie ist latent immer vorhanden, und das Verhalten richtet sich daran aus. 
Das Gebäude zeichnet sich durch seine Massigkeit und Wuchtigkeit aus. Nach außen repräsentiert es einen Herrschaftsbau, der jedoch nicht durch Prunk charakterisiert wird. Im Gegenteil wird die Geste der Herrschaft, die durch das Gebäude suggeriert wird, durch die Assoziationen mit militärischen Bauten, einer Kaserne oder einer Burg, erreicht. Das Gebäude stellt sich damit als Teil des Gewaltmonopols dar, dem sich die vorbeiziehenden und erst recht die eintretenden Subjekte zu unterwerfen haben. Die Intention des Gebäudes ist die Geste einer Machtasymmetrie und eine Adressierung der Subjekte als Subalterne. Dabei wird diese Unterwerfungsgeste auf der Binnenseite des Gebäudes noch verstärkt, indem zwei unterschiedliche Bewohner_innen suggeriert werden, deren Beziehung über eine Hierarchie geregelt ist. Die Beziehung stellt sich als Bewachungs- und Kontrollfunktion dar: Die Subalternen sind in diesem Bild nicht freiwillig hier.

Die ästhetische Umsetzung bedient sich der Merkmale, die für die Moderne bestimmend sind. Der Bau ist Sitz einer unpersönlichen Organisation, die das Prinzip von Rationalität ästhetisch repräsentiert. Sie tut dies in der Anlehnung an einen Verwaltungsbau und damit in der Verkörperung einer rationalisierten Ordnung. Sie bringt eine Ästhetik hervor, in der die massive Bauweise und die schmucklose Symmetrie die primären Gestaltungsmittel sind.

Wenn dem Gebäude ein Charisma attestiert werden kann, dann liegt dieses in einer Atmosphäre, die Akzeptanz durch Gefolgschaft verlangt. Das Gebäude stellt eine Herrschaftssicherung dar, indem es sich assoziativ an modernen (Kasernen) und vormodernen Herrschaftsbauten (Burgen) orientiert. Beides sind Gebäude, die der Durchsetzung von Herrschaftsinteressen dienen. Nach außen verkörpert das Haus in dieser Assoziation eine Schutzmacht und ein Gewaltmonopol.

Damit institutionalisiert sich nicht lediglich die Schule in der städtischen Siedlung. Das Schulhaus verkörpert ein Sinnbild einer staatlich organisierten zentralen Herrschaft.

\subsubsection{Raum(an)ordnungen - Pädagogik als serielle Praxis}

Als nächste und letzte Sequenz soll die Raumaufteilung im Schulhaus (Abb. 10). betrachtet werden. 
Es lassen sich dreierlei Ordnungsprinzipien ableiten. Zum Ersten kann konstatiert werden, dass das Gebäude aus drei Bauteilen besteht. Der größte, mittlere Teil beherbergt die großen Funktionsräume. Es ist der eigentliche Kern, sozusagen die heiligen Hallen des Gebäudes. Hinten und vorne sind diesem je ein Querteil vorgelagert, welche die horizontalen und vertikalen Erschließungen, die Räume mit sanitären Anschlüssen und - zumindest früher - die Garderoben beherbergen.

Zum Zweiten verweisen die Raumanordnungen darauf, dass das Gebäude als Doppelhaus konstruiert wurde. Es sind zwei gleichartige symmetrische Häuser unter einem Dach, die über je einen eigenen Zugang verfügen. Die Zugänge sind so angebracht, dass sie größtmöglich getrennt sind. Dass die Gebäude ursprünglich getrennt waren, darauf deutet die Lage der kleinen Verbindungen hin. Eine Durchlässigkeit ist von der Raumordnung her gedacht nicht vorgesehen.

Als Drittes soll die Raumordnung innerhalb der jeweiligen Häuser betrachtet werden. Hier befinden sich jeweils zwei Gebäudeteile, in denen ErschlieBungsteile mit Treppen und Funktionsräume wie Toiletten enthalten sind, die jeweils zweispännig den Kernteil umfassen.

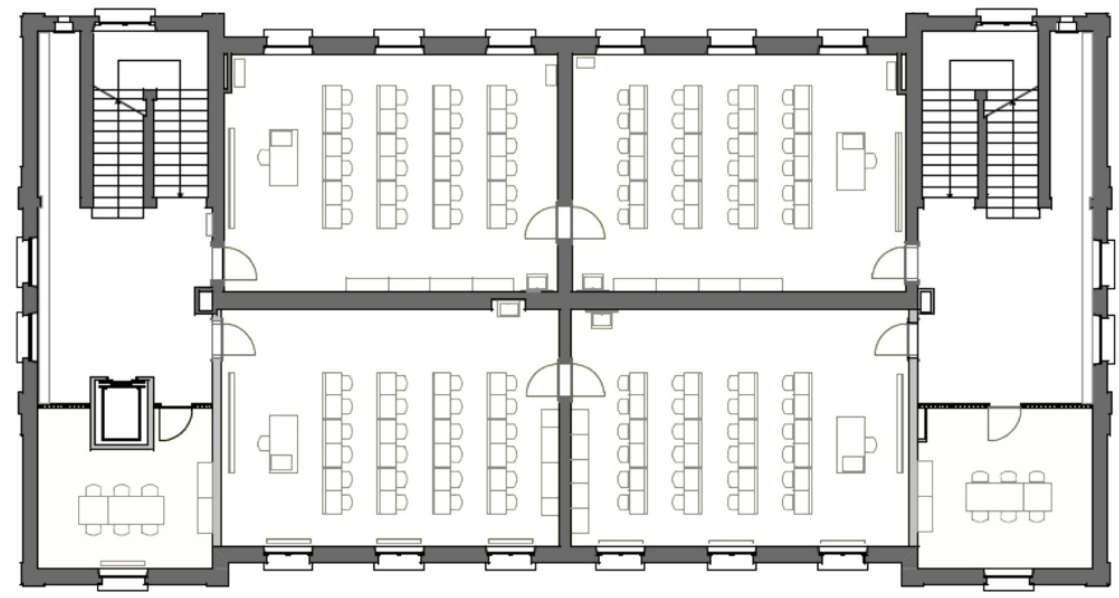

Abb. 10: Schulhaus Breitenrain, 2. OG. (C) Rykart Architekten AG, Bern 
Insgesamt kann festgehalten werden, dass das Gebäude einer strengen Einteilung folgt, die den Charakter eines Baukastensystems hat. Den Kern bilden die gleichartigen, normierten Klassenzimmer.

Das erste Ordnungsprinzip sind die drei Gebäudeteile. Vorne und hinten wird der mittlere Teil gerahmt. Die Rahmung wird dadurch unterstrichen, dass die beiden Teile über den Mittelteil hinausragen. In Analogie zu einem Bilderrahmen dienen die rahmenden Querteile dazu, den wesentlichen Teil - das Bild von außen abzugrenzen. Erst durch die Abgrenzung nach außen, die mit einem Ausschluss der Umgebung einhergeht, wird das Bild zu einer eigenen Welt der Suggestion. Die Rahmung hat den Effekt, den inneren Teil vom äußeren hermetisch abzugrenzen. Die Erschließungstrakte fungieren als eine rahmende Vorzone. Der innere Kern, die Räume pädagogischer Tätigkeiten, wird auf sich selbst bezogen. Schulräume sind damit weitestgehend frei von äußeren Einflüssen.

Dieser Umstand war im ursprünglichen Bauzustand noch expliziter, da die Garderoben in den Querteilen angebracht waren. Heute sind dort kleine Arbeitsräume untergebracht, und die Garderoben sind in die Schulzimmer verlegt. Das bedeutet, dass eine Schranke im Übergang vom Kind zum Schüler entfällt, indem persönliche Alltagsgegenstände und Kleider mit in den Schulraum genommen werden. Umgekehrt bedeutet es eine Verschiebung der ,innersten' InnenAußen-Abgrenzung in einen äußeren Bereich. Die explizite Trennung zwischen Unterricht und Nichtunterricht wird aufgehoben. Auch die Treppenhäuser und Erschließungsräume werden so mit pädagogischen Intentionen belegt. Die Gruppenräume ändern wenig an der Handlungsaufforderung des Gebäudes, die darin liegt, dass die Schüler_innen ohne Umweg schnellstmöglich an den ihnen zugedachten Platz gelangen sollen. Sie sind lediglich eine Aufweichung der funktionalen Trennungen in der Innen-Außen-Abgrenzung des inneren Kernbereichs und eine Umstrukturierung von reinen Erschließungsräumen zu pädagogisch nutzbaren Räumen.

Ein zweites Ordnungsprinzip ist in der gespiegelten Doppelung zweier Häuser zu sehen. Es ist augenfällig, dass es sich um ein Gebäude handelt, in dem zwei räumlich getrennte, aber symmetrisch angeordnete, d. h. funktional gleichförmige Einheiten untergebracht sind. Die Schule ist eine Organisation, die seriell aufgebaut ist, sie ist für seriell vonstattengehende Praktiken gebaut. Dasselbe Prinzip reproduziert sich in der kleinräumigen Raumeinteilung. Das Gebäude besteht mit Ausnahme der Erschließungsteile ausschließlich aus gleichartigen 
Räumen. Doch wird dies später noch zu diskutieren sein. Bis hierhin kann festgehalten werden, dass es sich um zwei gleichartige Raumorganisationen handelt, in denen - so ist zu schließen - eine gleichartige Praxis stattfindet. Es ist also eine Einheit von Gleichem und Differentem zugleich. Denn die Erschließungen sind so angebracht, dass die beiden Häuser größtmöglich getrennt sind. Die beiden Häuser können betreten werden, ohne dass von den Schüler_innen des jeweils gespiegelten Hauses Kenntnis genommen werden kann. Ein möglicher Kontakt, und sei es auch nur der Blickkontakt, wird vermieden. Zwar gibt es Verbindungen, die durch die Verbindungstüren sichtbar eingezeichnet sind. Die Raumaufteilung sowie die geringe Größe der Tür verweisen jedoch darauf, dass diese nicht als Durchgangswege für die Schüler_innen gedacht sind.

Aus dem Umstand, dass zwei gleiche Häuser unter einem Dach vorhanden sind, folgt, dass die Häuser für zwei gleich große Gruppen gebaut wurden. Es ist also zu fragen, nach welchen kategorialen Unterscheidungen differenziert wird. Da die Gebäudeteilung gleich große Gruppen nahelegt, ist eine Differenzierung nach Geschlecht die sparsamste Auslegung. Das Gebäude ist für die geschlechtergetrennte Unterrichtung von Mädchen und Jungen gebaut worden.

In der Bauart zeigt sich also eine gewisse Pragmatik, indem zwei Häuser unter einem Dach anstelle von räumlich getrennten Schulanlagen gebaut wurden. Diese Pragmatik kann auch so ausgelegt werden, dass die beiden Geschlechter trotz der Trennung als einander zugehörige und auf einander bezogene Einheit betrachtet werden. Allerdings ist in den öffentlichen - im Gegensatz zu den privaten und konfessionell ausgerichteten - Primarschulen in Bern faktisch nie geschlechtergetrennt unterrichtet worden. Geschlechtergetrennte Bildungswege waren dadurch vorgegeben, dass weiterführende Schulen im 19. Jahrhundert nur von Knaben besucht wurden. Aus der Architektur lässt sich schließen, dass der Diskurs über die Koedukation ein zentrales Politikum war. Die Gebäude wurden so erbaut, dass jederzeit auf einen geschlechtergetrennten Unterricht umgestellt werden kann. Diese Konfliktlinie, die im Wesentlichen eine Konfliktlinie zwischen Kirche und Staat war, war lange Zeit nicht entschieden. Denn in der Stadt Bern wurden sämtliche Schulgebäude, die zwischen 1850 und 1880 gebaut wurden, mit zwei Treppenanlagen ausgestattet. Somit wäre ohne großen baulichen Aufwand eine Geschlechtertrennung möglich, obwohl diese faktisch nie vollzogen wurde (Gurtner 1997: 17 f.). 
Es objektiviert sich in der Architektur ein Umstand, der wohl auch für Planung und Konzeption des Schulhauses Breitenrain angeführt werden kann. Geiss (2012: 77) weist darauf hin, dass die Bildungsverwaltungen ihren Einfluss zwischen den Konfliktlinien von Staat und Kirche im 19. Jahrhundert sukzessive ausbauen konnten. Damit gewannen die subsidiär übergeordneten Bildungsverwaltungen an Einfluss auf die Ausgestaltung der Schulen, obwohl die Gemeinden die materielle Hauptlast des Verstaatlichungsprozesses im Zuge der Nationalstaatskonsolidierung wie zum Beispiel die Baukosten trugen. Das Schulhaus Breitenrain stellt nicht nur eine herrschaftliche Staatlichkeit dar, sondern es repräsentiert diese in einer ganz spezifischen Form als subsidiär funktionierende Behördenstruktur, die auf den Prinzipien einer rational organisierten Bürokratie beruhen. Schulen sind damit auch Agenturen des jungen Nationalstaates und Verkörperungen einer funktionierenden Bildungsverwaltung sowie generell einer bürokratisch organisierten Expertenherrschaft. Dies drückt sich offensichtlich auch in der Architektur der Schulhäuser aus.

In der horizontalen Gliederung ist die Raumordnung des Gebäudes dadurch charakterisiert, dass der funktionale Erschließungsteil minimalistisch raumsparend konzipiert ist. Gleich hinter der Tür trennen sich die Wege. Es gibt keinen gemeinsamen Innenraum beziehungsweise ist das, was als Residuum von gemeinsamem Innenraum bleibt, vorwiegend Verkehrsfläche. Der Übergang zwischen Pause und Unterricht ist so kurz wie möglich.

Die Erschließung ist zweispännig organisiert, d. h. pro Stockwerk werden zwei gleichartige Räume erschlossen. Das Resultat ist eine Aufteilung in mehrere einheitliche Klassenzimmer. Wenn man die beiden Gebäude auseinanderdenkt, dann findet sich pro Haus eine Raumstruktur vor, die man als Urhütte oder Prototyp des Gebäudetyps Schulhaus denken kann. Es ist eine zweispännige, symmetrische Duplexbauweise, mit gleichartigen Klassenräumen. ${ }^{32}$ Diese können im Prinzip fast beliebig gestapelt werden. Der Vorteil dieser Bauweise ist, dass in der Breite beliebig viele Räume angehängt werden können. So gibt es Schulhäuser, die ohne Weiteres um fünfzig Meter in irgendeiner Richtung verlängert werden könnten, ohne dass befürchtet werden muss, den architektonischen Eindruck mehr als höchstens quantitativ zu verändern (siehe Kemnitz 
2008: 253). Beim Schulhaus Breitenrain ist dies nicht so, gerade weil das Schulhaus aus zwei Gebäuden kombiniert wurde, die einander gegenübergesetzt sind. Das Haus resultiert aber aus einer Architektur, die aus einem variablen Baukasten identische Einheiten aneinanderreiht und stapelt. Das Schulhaus Breitenrain scheint weniger als Komposition eines Ganzen entstanden zu sein, wie dies für die vormoderne Architektur wesentlich ist. Sie ist eine Einheit - beziehungsweise genau genommen eben eine Zweiheit - aus kombinierten und gestapelten normierten Räumen, die als Doppelhaus einander gegenübergestellt sind. Das Schulhaus Breitenrain ist nach einem Planungs- und Bauprinzip hergestellt, das auf der Anordnung von normierten gleichartigen Räumen beruht. Dies hat architekturhistorisch, aber auch bildungstheoretisch weitreichende Bedeutung.

Einheitlich normierte Klassenräume sind für eine Unterrichtung der Schüler_innen in der Jahrgangsklasse ausgerichtet. Diese war in der ersten Hälfte des 19. Jahrhunderts nicht die dominante Form. Im Gegenteil war es ein heterogener Diskurs, der nicht nur, aber hauptsächlich in der Differenz zwischen Fachklassen und Jahrgangsklassen geführt wurde. Die Jahrgangsklassen haben sich laut Scholz und Reh darum als „Muster schulischer Organisation universalisiert“ (Scholz \& Reh 2016: 94), weil es die Organisationsform ist, die dem Leistungsprinzip und der Allokationsfunktion von Schule am besten dient (ebd. S. 109).

Normierte Klassenzimmer sind Ausdruck einer Organisation, die verwaltungstechnisch operiert. Verwaltungstechnisch erlauben identische räumliche Einheiten eine flexiblere organisatorische Ein- und Umteilung der Schüler_innen in den gegebenen konstanten Raumgrößen der Klassenzimmer. Das normierte Schulhaus kann also als Resultat einer zentralen, bürokratisch agierenden Organisation gelesen werden, obwohl oder vielleicht gerade weil die Bildungsverwaltungen die Schulbauten nicht planen, sondern lediglich Empfehlungen abgeben. In dieser Lesart kann ein weiteres Argument starkgemacht werden, welches die bisher explizierte Fallstruktur schärft.

Das Schulhaus Breitenrain repräsentiert die Prinzipien, die sich über Jahrzehnte als Verkörperungen eines bürokratischen Verwaltungsstaates routinisiert haben. Zudem können Schulen als Agenturen der Bildungsverwaltung und damit auch als Agenturen einer funktionierenden Behördenstruktur gelesen werden. Das durchrationalisierte und normierte Schulhaus Breitenrain kann als zunehmender Einfluss einer subsidiär übergeordneten Bildungsverwaltung gesehen werden. Denn der Gebäudetyp Schulhaus ist aus Normierungsbestrebungen ent- 
standen, wie sich dies schon sprachlich in den ,Normalien" (Gohl 1888; Salvisberg 1870) manifestiert. Der Sinn der Normalien war es, den Gemeinden variabel umsetzbare Pläne für eine - kantonal geregelte - einheitliche Schulpädagogik zu liefern (ausführlich Helfenberger 2013). Damit haben Schulhäuser nicht nur einen Einfluss auf die Ausdifferenzierung der Schule zu einer uniformen Institution. Sie sind auch Vorreiter, die einen wesentlichen Einfluss auf die Normierung von Gebäuden und einer Architektur haben, die auf Normen und Standards einerseits und einer einheitlichen Architektursprache andererseits beruht. Dies, obwohl oder vielleicht gerade weil die ,Normalien“ so konzipiert waren, dass sie auf standardisierten Klassenräumen als Kernzellen beruhten. Denn der Vorteil dieser normierten Bauweise ist, dass diesen Gebäuden ein fast beliebiger Mantel umgehängt werden kann. ${ }^{33}$ Dieses variable System, das mit einem beliebigen Mantel kombiniert werden kann, ist mit ein Hauptgrund, dass dieses Gebäudeprinzip über Jahrzehnte hinweg als Prototyp der meisten Schulhäuser im 19. Jahrhundert Bestand hatte (vgl. H. Schneider 1969).

\subsubsection{Klassenzimmer als Grundzellen eines monofunktionalen Konzeptes in seriellen Räumen}

Das Haus besteht neben den Erschließungsteilen im Wesentlichen aus sechzehn gleichartigen Räumen, wovon die vier Räume im Untergeschoss aufgrund der Lichtverhältnisse differenziert betrachtet werden müssen. Die zwölf oberirdischen Räume verweisen in ihrer Gleichförmigkeit auf eine gleichartige, das heißt serielle Praxis. Die Räume sind je ca. 60 Quadratmeter groß. Sie sind quasi die „Grundzelle des Klassenzimmers“ (Perlick 1969: 50), die sich in dieser Größe und Form bis heute erhalten hat. Ein Grund dafür wird sein, dass dieser Räumlichkeit gewisse Grundkonstanten eingeschrieben sind, die für die öffentliche Schule eine wesentliche Konstante darstellen. Welches sind die Strukturprinzipien, die den Raum auszeichnen? Die Schüler_innen-Zahl kann es nicht sein. Die

33 Ein umgekehrtes Prinzip verfolgt die Bauweise des Bauhauses (vgl. Steets 2015: $118 \mathrm{ff}$.). Diese geht nicht von normierten Räumen, sondern von normierten Bauteilen aus, die variabel zu theoretisch unendlich vielen Gebilden zusammengefügt werden können. 
Klassenräume haben sich in ihrer Grundstruktur nur unwesentlich verändert, während die Anzahl der Schüler_innen, die darin unterrichtet werden, sich auf einen Bruchteil verringert hat.

Die unterschiedslosen Räume sind speziell für ein Gebäude. Wohnhäuser, Gewerbehäuser, Museen, aber auch Bürobauten bestehen aus unterschiedlichen Räumen, die funktional für verschiedene Praktiken gedacht sind: fürs Schlafen, Kochen, Essen, Arbeiten, als Verkehrsflächen usw. In der heutigen Nutzung ist diese Differenzierung beim Schulhaus Breitenrain offensichtlich auch gegeben. Wie auf den Plänen ersichtlich ist, gibt es eine Bibliothek, eine Tagesschule, ein Lehrerzimmer und Klassenzimmer usw. Aus den Raumformen und -kubaturen muss geschlossen werden, dass dies Umnutzungen sind. Dies tritt noch verstärkter hervor, wenn wir bedenken, dass auch die Toiletten erst 1926 errichtet wurden. ${ }^{34}$ Das Breitenrain-Schulhaus ist - abgesehen von den Erschließungen monofunktional konzipiert. Es erwartet von den Nutzer_innen, sich diszipliniert an ihren jeweiligen Platz zu begeben und innerhalb dieses Platzes eine monotone, offensichtlich serielle Praktik auszuüben.

Die spezifischen Merkmale eines Raums mit einer Fläche von 60 Quadratmetern ist eine Raumgröße, die Überblick gewährleistet. Jeder Punkt im Raum erlaubt den gesamten Raum im Blick zu behalten. Für das Publikum ist dies wesentlich. Es ist für die Kontrolle durch die Lehrperson genauso zentral. Größere Räume, zum Beispiel Hörsäle, sind in ökonomischer Hinsicht wesentlich sparsamer. Sie bedürfen aber einer disziplinierten Klientel. Andererseits drückt schon der Name aus, dass es Hörsäle und keine ,Sehsäle“ sind, in denen Vorlesungen gehalten werden. Das Klassenzimmer in dieser Form ist der größtmögliche Raum, in dem eine Vermittlung über eine visuelle Veranschaulichungsfläche stattfinden kann. Es ist ein Raum, in welchem zentralperspektivisch die Anschauungsfläche von überallher ersichtlich ist. ${ }^{35}$

Eine Veranschaulichungsfläche ist für den sogenannten Frontalunterricht konstitutiv und vice versa. Die Blickweiten sind dabei in beide Richtungen we-

34 Darin reproduziert sich auch eine Sinnstruktur, welche die Adressierung der Schüler_innen als unpersönliche Rollenträger_innen sieht. Nicht mal für physiologische Vorgänge körperlicher Ausscheidungen wurden Räume geschaffen, was den Schüler_innen eine ungeheure Disziplinierung des eigenen Körpers abverlangte. 
sentlich. Die Raumgröße in einem ,Sehraum“ ist analog zum ,Hörraum“ gestaltet. Von jeder Stelle aus kann der Raum überblickt werden, und die Distanzen sind so, dass der Hörende den Sprechenden auch in der entferntesten diagonalen Anordnung noch versteht, wenn dieser in normaler Lautstärke spricht. Die Limitierung beträgt auch hier unwesentlich mehr als bei der Sichtweite. Die Akustik ist aus der Senderperspektive weniger limitierend, da die Akustik durch ansteigende Räume enorm optimiert werden kann, wie dies Hörsäle, aber auch die antiken Theater veranschaulichen. Wenn die Akustik jedoch auch als Mittel der Überwachung und Kontrolle der Schüler_innen gesehen wird, dann ergibt sich erneut eine Limitierung der Raumgröße, die ungefähr diesen 60 Quadratmetern entspricht. Dieser Raum ist gerade noch visuell und akustisch überwachbar. Der Klassenraum hat in dieser Größe akustisch gesehen eine gewisse panoptische oder besser gesagt panakustische Funktion. Alles kann gehört werden, ohne dass für den Sprechenden erkennbar ist, ob aktiv zugehört wird.

Betrachten wir erneut die konkreten Räumlichkeiten. Die horizontale zweispännige Erschließung der Klassenräume hat implizit eine eindeutige Ausrichtung der Klassenzimmer zur Folge. Dies ist eigentlich nicht zwingend, es stellt sich aber in allen Klassenräumen so dar und reproduziert die Struktur der Beziehung zwischen ,Riesen " und ,Zwergen', die an der Fassade rekonstruiert wurde und die sich nun explizit als Beziehung zwischen Lehrer_innen und Schüler_innen manifestiert. Die eindeutige Raumaufteilung findet sich an vielen Details: Als wichtigstes Element ist sicherlich die Wandtafel als Anschauungsfläche zu nennen, die eine ,Vorderseite" des Raums festlegt. Sie bestimmt die Ausrichtung der Schüler_innen und unterteilt den Raum in zwei Zonen: die Zone der Lehrperson und die weitaus größere Zone der (sitzend-immobilen) Klasse. 


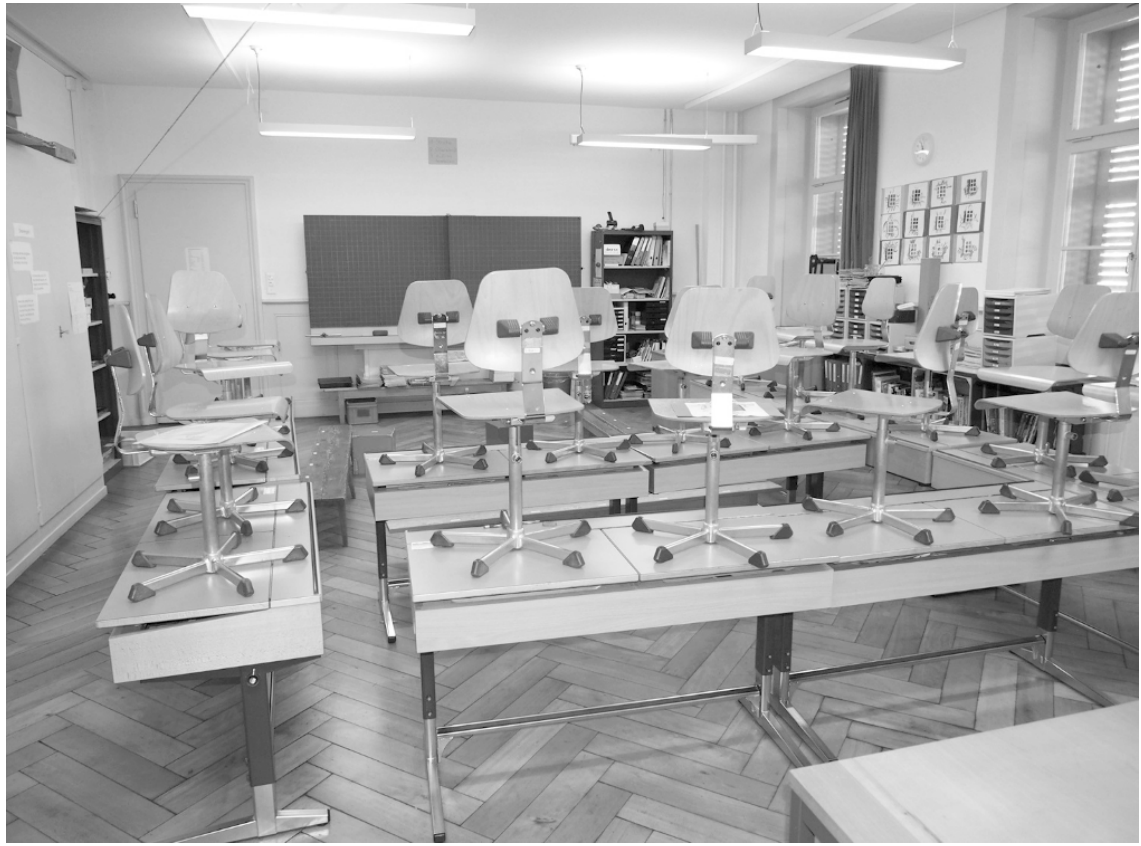

Abb. 11: Schulhaus Breitenrain. Klassenzimmer

Dies kann man sich am einfachsten klarmachen, wenn man kontrastiv an Kindergärten denkt, für welche diese Struktur nicht gilt. Mit der Zweiteilung durch die Anschauungsfläche wird der Raum in zwei Bereiche aufgeteilt, was der Struktur von Bühne und Publikum entspricht. Die Bühne oder analoge räumliche Settings sind mit Goffman (1994) gedacht ein Ort, wo platform performances stattfinden. Für diese sind Interaktionsordnungen bezeichnet, bei denen eine klare Richtung der Wahrnehmung und der Interaktion vorgegeben sind.

Als weitere gemeinsame Merkmale der Klassenzimmer sind die Anschauungsflächen an derselben Seite wie die Erschließungen angebracht. Es sind die Eingänge, die darüber befinden, wo vorne und wo hinten ist. So ist nicht nur die Raumordnung einseitig auf die Lehrerzone ausgerichtet. Im Unterschied zu einem Theater, in dem die Wege der Schauspieler_innen und des Publikums geschieden sind, ist hier das Publikum Teil der Inszenierung. Der Raum wird über die ,Bühne' betreten. Theoretisch ist zwar ein Betreten von hinten möglich. Dies 
impliziert aber, dass der Betretende das Haus von der anderen Seite her betreten hat und zuvor die Bühne und das Publikum eines anderen Klassenzimmers durchquert. Es verbleibt die Feststellung, dass der Raum nicht betreten oder verlassen werden kann, ohne die Lehrperson zu passieren. Die Lehrperson hat die Kontrolle darüber, wer den Raum betritt und verlässt.

Für welche Publikumsordnung ist der Raum gedacht? Wenn man die Pläne betrachtet, sind einheitliche Bestuhlungsordnungen ${ }^{36}$ eingezeichnet. Die Möblierung ist in vier Reihen aufgebaut, die Blickrichtung geht in Richtung der Treppenhäuser. Dort ist ein etwas größeres Pult eingezeichnet, das eine umgekehrte Blickrichtung hat. Die Publikumsanordnung ist jeweils auf die Lehrperson ausgerichtet. Damit sind die Schüler_innen ausschließlich auf die Lehrperson ausgerichtet und nehmen, sofern sie in den hinteren Reihen sitzen, die Mitschüler nur von hinten war. Eine Beziehung zwischen den Schülern ist in dieser Sitzordnung nicht vorgesehen.

Historisch gesehen wurde diese Raumaufteilung und Anordnung durch die Subsellien festgeschrieben (Hnilica 2010). Die feste Installierung von Schulbänken und -pulten hat Präzedenzen geschaffen, die sich als spezifische Raumordnung in den Raum so tief eingeschrieben haben, dass sie auch heute noch die Raumordnung prägen. In dem Raumsetting des Gegenübers von Schüler_innen und Lehrperson wird mit den Subsellien fixiert, dass die Schüler_innen im Klassenzimmer ,immobil ' gedacht werden. Sie sind auf ihren festen Plätzen, während die Lehrperson mobil ist. Das antagonistische Gegenüber von Schulklasse und Lehrperson strukturiert eine spezifische Interaktionsform und Interaktionsordnung, die einer festen und strengen Dramaturgie unterliegt. Wenn die Abbildungen der Klassenräume betrachtet werden, scheint sich die Struktur der Subsellien in gewisser Weise im Raum erhalten zu haben. Raumaufteilung und Ausrichtung sind mit gewisser Variation gleich geblieben. Für die Ausgestaltung eines Arbeitsbündnisses zwischen Lehrperson und Schüler_innen bedeutet dies, dass die Sitzordnung ein Arbeitsbündnis vorstrukturiert, indem die Klasse und weniger der/die einzelne Schüler/Schülerin dominant als Adressat der Unterrichtspraxis

36 Anzufügen ist, dass die Möblierung streng genommen von der Architektur zu unterscheiden ist. Die Möglichkeiten der Möblierungen werden aber doch auch durch die Architektur strukturiert. Zudem wird Architektur immer schon mit einer spezifischen Idee der Möblierung konzipiert. Deshalb wird die Frontalbestuhlung in diesem Falle als Teil der Architektur betrachtet. 
anzusehen ist. Der Einzelne wird innerhalb des Gesamten adressiert. Das heißt, dass in der Frontalbestuhlung die Sitzordnung auch zu einer Rangordnung wird. Die Anordnung in Reihen und Bänken errichtet zwangsläufig ein Ordnungssystem, eine serielle Rangfolge. Das Schulmobiliar konstituiert als Teil fester Architektur auch eine Rangfolge der Schüler_innen. Die Position, an der sich die Schüler_innen jeweils befinden, impliziert einen unterschiedlichen Rang und Status. Dies gilt sowohl unter der Prämisse, dass eine feste Platzzuteilung stattfindet, als auch dann, wenn dies nicht der Fall ist. In letzterem Falle findet eine Selbstselektion statt, welche Plätze eingenommen werden. Sowohl den Schüler_innen als auch den Lehrpersonen ist implizit klar, wo die Plätze sind, die an bestimmte Rollen gekoppelt sind. Zielsicher suchen sich Schüler_innen und Studierende Sitzplätze, die den Rollen entsprechen, die sie einzunehmen gedenken. Diese Regeln sind zum Teil fix an Raumordnungen oder gewisse Gegenstände gebunden. Sie sind aber auch relational abhängig davon, wer schon im Raum sitzt.

Mit den Subsellien sind wir methodisch gesehen in einer Grauzone. Die Möblierung wird streng genommen nicht als Teil der Architektur betrachtet. Umgekehrt waren die Subsellien fest mit dem Gebäude verbunden und damit Teil der Architektur. Viel wichtiger jedoch scheint mir, dass sich durch die Subsellien die Sitzhaltung als schulische Grundhaltung festgeschrieben hat. Sie haben die Möglichkeiten, wie ein Klassenraum auszusehen hat und Unterricht vonstatten gehen soll, bis heute maßgeblich beeinflusst. Aber nicht nur die Architektur ist davon betroffen. Die Sitzhaltung als schulische Grundhaltung hat selbstredend massive Auswirkungen auf die Art des schulischen Wissens. Dieses liegt damit zwangsläufig in einer abstrahierten, für die Veranschaulichungsfläche konzipierten Form in Form von Schaubildern, Sammlungen usw. vor.

Das Argument ist, dass sich in der Sitzordnung eine sukzessive Internalisierung von Normen und Verhaltensdispositionen zeigt, die in der Entstehungszeit des Schulhauses Breitenrain durch physischen Zwang beziehungsweise durch disziplinarische Zurichtung erreicht wurden. Die mit den Subsellien explizit erzwungenen Handlungs-, aber auch Aufmerksamkeitsdispositionen und Interaktionsformen sind heute den Gegenständen eigen. Schon der Griff der Lehrperson zur Kreide ist für die Schüler_innen mit Verhaltensaufforderungen verbunden. Dies gilt noch viel expliziter für das Aufrufen eines Schülers an die Wandtafel. Die Zuschreibung bzw. die Kopplung von Verhaltensdispositionen an Gegen- 
stände ist nur über die Normierung und Institutionalisierung möglich. Im normierten Klassenzimmer mitsamt seinen Einrichtungen kulminieren die Strukturprinzipien eines asymmetrischen Unterrichts zwischen Lehrperson und einer Gruppe, die als Gruppe homogener Schüler_innen adressiert werden.

Zusammenfassend kann gesagt werden, dass die Architektur ein Setting herstellt, in dem die Schüler_innen primär rollenförmig angesprochen werden. Die ganze Architektur ist in ihrer Funktionalität, ihrer Adressierung und ihren repräsentierten Sinnstrukturen darauf gerichtet, die Individuen gerade nicht in ihrer Individuiertheit zu adressieren. Im Gegenteil ist alles auf die Tilgung des Individuellen ausgelegt. Die Subjekte werden als Rollenträger adressiert.

Das Schulhaus Breitenrain stellt eine Architektur dar, die für eine Unterrichtung gebaut wurde, die als extern initiierte Unterweisung durch eine Lehrperson angesehen werden kann. Das Haus ist errichtet in Räumlichkeiten, die sich durch ein Raster auszeichnen, welches auf eine serielle Unterrichtung gerichtet ist. Die Räume bilden ein Setting, welches eine ganz spezifische Interaktion und eine Interaktionsordnung konstituiert. Dieses ist asymmetrisch, und es gibt einen quasi natürlichen Fluss des Wissens, vom vereinten Wissen vorne im Raum (markiert mit Tafel, Materialien, Regalen, Lehrerpult) zu den Schüler_innen, die implizit oder explizit nach einer bestimmten Rangordnung im Raum verteilt sind. Die Schüler_innen werden rollenförmig adressiert, indem ihnen ein gemeinsamer Wissenskorpus vermittelt wird. Nicht das zu vermittelnde Wissen oder die Bildungsinhalte werden aufgeteilt, sondern die Schüler_innen werden aufgeteilt nach dem Passungsverhältnis zum Wissen oder zur Art der Wissensvermittlung. Die Architektur bietet keinerlei Räume, in denen ein Rückzug in eine gewisse Privatheit vollzogen werden könnte.

Eine Strukturtransformation lässt sich an den heutigen Umnutzungen erkennen. Das Erdgeschoss wurde umgewidmet zu Räumen, die für selbsttätige Praktiken der Schüler_innen gedacht sind. Es sind Räume, die ein Angebot und eine Anregung darstellen, die selbsttätig angeeignet werden müssen. Während sich im seriellen Raum eine Deutung von Sozialisation als von außen über Disziplin gesteuert darstellt, manifestiert sich in einer Bibliothek und in einem Medienraum eine Deutung dahingehend, dass Bildung selbsttätiger Aneignung bedarf.

Das Schulhaus verkörpert die eigentliche Wissensvermittlung. Es ist etwas reduktionistisch-metaphorisch gesprochen das ,Gehirn“ des Schulareals. Es verkörpert eine strikte Trennung von rein kognitiven Tätigkeiten, die im Klassen- 
zimmer vonstattengehen, und praktischen Tätigkeiten, die nur durch die eigene Herstellung erlernt werden können. Es kann also erwartet werden, dass sich außerhalb des ,Gehirns' die zusätzlichen Funktionen eines ,Organismus ‘ feststellen lassen. Als intentionales Ziel des Gebäudes wurde eine Adressierung der Schüler_innen als Soldaten rekonstruiert. In der ursprünglichen Intention des Gebäudes wäre also anzunehmen, dass sich die weiteren Elemente in die Sozialisation zu einem militärischen Subjekt einreihen.

\subsubsection{Pädagogisch intendierte Sozialisation über Leib- und Körpererfahrung im Schulsport}

Es wurde festgehalten, dass über die Hälfte der Fläche in Form von Turnhalle, Garderobe und blau eingefärbtem Sportplatz durch den Schulsport belegt ist. Der Sport ist ein wesentliches Element der Schule Breitenrain, sowohl in seiner Funktion als Unterricht als auch als Nichtunterricht: Die Bauten und Flächen des Schulsports nehmen annähernd die Hälfte des verfügbaren Raums ein. Der Platz ist explizit markiert, wobei die Farbe nicht nur auf den Sportplatz beschränkt ist, sondern auch die Überreste des Innenhofs bestimmt. Diese farbliche Markierung und die räumliche Abgrenzung konstituieren zwei Hälften: im südlichen, vorderen Teil eine Zone, in der das Haupthaus dominant ist, und im nördlichen, hinteren Teil eine, in der der Sport eine Binnenfunktion erfüllt. So muss man für den Schulsportplatz eine Doppelfunktion annehmen. Er ist einerseits für den (Turn-) Unterricht konzipiert. So ist das Spielfeld Teil der Infrastruktur für den Unterricht. Andererseits ist er als Ort des Nichtunterrichts also auch in den Pausen zugänglich.

Aus den Linien, den Toren und den Basketballkörben ist er eindeutig als polysportive Sportplatz zu identifizieren. Sportplätze beziehungsweise Anlagen zur sportlichen Betätigung sind ein zentraler, sichtbarer Code von Schulanlagen. Sie können als notwendige, wenn auch nicht hinreichende Bedingung für die Erken- 
nung von Schulanlagen gelten. ${ }^{37}$ Auffallend ist die Färbung des Platzes, in der Regel werden Außenbeläge neutral gehalten. Sie sind nur dort farbig markiert, wo sie explizit eine Signalisationswirkung entfalten, etwa bei Radwegen oder Spielstraßen, bei denen Autofahrer_innen gewarnt werden. Sportplätze sind in der Regel in einem rostroten Farbton gehalten, was aus dem Material, dem Tartan, resultiert.

Das leuchtende Azurblau des Platzes ruft die Assoziation einer Wasseroberfläche auf, etwa einer blauen Lagune oder einem Swimmingpool. Als Sinnstruktur steht der Swimmingpool für Außeralltäglichkeit und ein Enthobensein von Alltagsroutinen in Form von Ferien und Freizeit. Der blaue Platz vereint damit die Funktionalität eines wettkampf- und körperbetonten Mannschaftssportes mit der ästhetischen Andeutung eines außeralltäglichen, sorgenfreien Lebens. Dem Schulhausplatz wird damit die Sinnstruktur von Freizeit eingeschrieben. Auch Sport ist zunächst Außeralltäglichkeit in dem Sinne, dass er außerhalb ökonomischer Tätigkeiten steht. Dem Sport wird über die Farbe die Sinnstruktur einer intrinsischen Motivation aus sich heraus unterstellt.

Sport ist ein eigenlogisches Spiel, nach je eigenen konstitutiven Regeln, die nur in dem räumlich-zeitlich-sozialen Setting gelten, die das Spiel ausmachen. Sport hat eine spielerische und eine wichtige sozialisatorische Komponente. Dies zeigt sich hier an der blauen Markierung und dem Feld als Ort: Über Markierungen werden gewisse Bewegungsabläufe vorgegeben und soziale Verhaltensweisen initiiert und konstituiert. Ein Spielfeld ist ein zentraler Ort, wo Regeln einverleibt werden.

Für die Schule Breitenrain scheint wesentlich, dass der Sportplatz sowohl Teil von organisierter Bildungsveranstaltung als auch ein gestaltetes Artefakt des Nichtunterrichts ist. In Letzterem stellt er eine architektonische Geste, eine Einladung zur selbsttätigen Sozialisation dar. Diese besteht in einer spezifischen Form, nämlich derjenigen des Spiels in Form eines Wettkampfs von mindestens zwei Gruppen. Dies wird noch eingehend zu diskutieren sein.

37 Als hinreichend können sie in Kombination mit großen Gebäuden betrachtet werden, die die Strukturprinzipien einer seriellen, auf eine kollektive Klassengröße bezogene Räumlichkeiten darstellen. 
Zunächst soll aber die Struktur thematisch sein, mit der der Schulsport in Bezug auf das Gesamt der Schule durch die Architektur verbunden wird. Festgestellt wurde, dass der Turnunterricht beziehungsweise der Schulsport räumlich von den schulischen Bildungsaufgaben geschieden wird. Der körperlich-leibliche Bildungsprozess wird von den geistigen oder nichtleiblichen Bildungsprozessen räumlich getrennt und damit funktional nach außen vereint und nach innen entzweit. Vereint sind sie darin, dass die Anlagen und Artefakte des Schulsports ein zentraler Code der öffentlich-rechtlichen Schule darstellen. Sie sind für diese konstitutiv. In der Binnenbetrachtung sind sie geschieden, wobei die beiden Bereiche aufeinander verwiesen sind. Geistige Bildung und Leibesertüchtigung sind je hälftig aufgeteilt. Unterricht bedingt den Nichtunterricht als Kompensation zum geistigen Bildungsprozess.

Damit zeigt sich eine Sinnstruktur, die Sport, Spiel und den Turnunterricht als Teil einer Reproduktionsfunktion sieht. Nichtunterricht oder Pause als Ort naturwüchsigen Verhaltens und von Sozialisationsprozessen dient der (Wieder-) Herstellung einer körperlich-geistigen Verfassung, die als Voraussetzung für die eigentlich pädagogisch-didaktischen Bildungsveranstaltungen steht. Dabei ist zeitlich und räumlich das einzelne Subjekt in seiner Privatheit für die Reproduktion zuständig. Die Wahl der körperlichen Betätigung (z. B. Klettern oder Ballspiel) sind öffentlich einsehbar, aber in der Verantwortung des jeweiligen Subjekts in den Pausen bzw. der ,Freizeit‘. Diese Trennung von Körper und Geist, oder genauer von körperlicher und geistiger Bildung, manifestiert sich in der Architektur als ein charakteristisches Merkmal der öffentlich-rechtlichen Schule.

Körperliche Bewegung und Sport(-Unterricht) ist ergänzende Antipode zum Unterricht. Er ist notwendige Bedingung für Unterricht, liegt aber außerhalb der eigentlichen Bildungsziele. In diesem Sinne lässt sich die räumliche Einheit der Architektur der Sportanlagen in ihrer Doppelfunktion von Bildungsveranstaltung und Raum intrinsischer Sozialisationsprozesse auflösen. Dem Turnunterricht und der Bewegung wird eine kompensatorische Funktion zugewiesen, die für die Schule konstitutiv ist. Diese Kompensation verläuft sowohl naturwüchsig in der Peer Group in den Pausen. Sie verläuft aber auch im organisierten Turnen unter Anleitung der Lehrperson.

Im Folgenden wird die Funktionalitäten der sichtbaren Sportplätze detaillierter in Bezug auf die sozialisatorischen Wirkungen betrachtet, die dem sportlichen Spiel eingeschrieben sind. Das blaue Feld ist ein normiertes Allzweckspiel- 
feld. Es kann sowohl als Basketball- und Handballfeld oder auch als Fußballplatz genutzt werden. Die Gemeinsamkeit dieser Sportarten besteht darin, dass es Mannschaftssportarten sind. Was ist die Besonderheit eines Teamsports? Worin ist der sozialisatorische Prozess innerhalb des Sports zu sehen? Voraussetzung für das Funktionieren einer Mannschaftssportart ist, dass sich die Subjekte als Teil der Mannschaft verstehen und damit identifizieren. Um zugehörig und Mitglied einer Gruppe zu sein, muss man sich selbst in den Dienst der Gruppe stellen, was auch eine Unterordnung beinhaltet. Man muss sich einer kollektiven Entität einordnen. Mannschaftsspiele funktionieren arbeitsteilig; es sind unterschiedliche Rollen vorhanden. Das einzelne Subjekt muss die Rolle des Torwarts, des Liberos usw. übernehmen. In einer sozialisatorischen Perspektive ist der Teamsport also ein Ort, wo die Rollenübernahme über die körperliche Erfahrung internalisiert wird.

Spiele werden von Regeln geleitet, wobei mit Regeln nicht lediglich die Spielregeln gemeint sind. Spiele dienen der Einübung von Regelwissen, die über das Spiel hinaus gelten. Regelverletzungen ziehen Sanktionen nach sich. Im Spiel wird regelgeleitetes, normativ konformes Verhalten eingeübt. Durch die Routinisierung der Regeln und Normen durch ständiges Einüben werden diese einverleibt. Dabei werden vor allem gesellschaftlich gültige Regeln inkorporiert, die im hochgradig kodifizierten Sport auf unterschiedlichsten Ebenen vorliegen: in Form von gültigen Regeln wie Fairness oder Wettbewerb, in Geschlechterrollen sowie damit einhergehenden Körperidealen und Formen des Körpereinsatzes etc.

Das Spielfeld steht für eine Sozialisationsinstitution, in welcher die gesellschaftlichen Regeln über körperliche und sinnliche Erfahrung einverleibt werden. Dabei ist der Sport eine Institution, die weit über die naturwüchsige Bewegungssozialisation durch Rennen, Hüpfen usw. hinausreicht, indem äußere gesellschaftliche Regeln und Werte mittransportiert werden. So findet im Sport eine spezifische Leib- und Selbsterfahrung statt, die in der Einübung, Erprobung und Ertüchtigung von Ausdauer, Grenzerfahrungen und physischer Leistungsfähigkeit liegt. Sie alle sind Formen eines Körpereinsatzes, der (Selbst-)Disziplin, unbedingten Willen und Körperkontrolle voraussetzt oder wo diese eingeübt und einverleibt werden. 
Um besonders erfolgreich zu sein, müssen die Regeln und die Körperkontrolle so weit routinisiert werden, dass sie zur unbewussten (Re-)Aktionen, zur eigenen ,Natur', werden.

Eine für den Schulsport nicht unwesentliche Sinnstruktur liegt darin, dass der Sport mit einer gewissen Leidensbereitschaft einhergeht. Es geht darum, sich selbst unter den Aspekten von Leistung, Grenzerfahrung der Leistungsfähigkeit und dem relativen Vergleich mit anderen im Wettbewerb wahrzunehmen und eine Leidensbereitschaft unter einen ,höheren', kollektiven Sinn unterzuordnen. Damit wird eine weitere Perspektive angesprochen, die im Zusammenhang mit dem Schulturnen noch eingehend zu betrachten sein wird.

Sport und Wettkampf wurden bis hierhin primär unter sozialisatorischen Aspekten diskutiert, die für den Bildungsprozess der Individuen entscheidend sind und die das Autonomiepotenzial der Subjekte unterstützen beziehungsweise entfalten. In dieser Sinnstruktur zeigt sich der Schulsport im Schulhaus Breitenrain in den Formen eines Teamsportplatzes und in der Deutung, dass dies weitestgehend selbsttätig durch die Sozialisanden zu geschehen hat. Dabei ist bezeichnend, dass das Spiel auf dem Pausenhof und im Unterricht primär innerhalb der Peer Group vonstattengeht. Sie erfolgt mehrheitlich selbsttätig. Es ist aber anzufügen, dass die Intention im Wesentlichen über ein Artefakt hergestellt wird. Es ist eine äußerliche, durch Architektur hergestellte Intention, dass auf dem Schulhof Breitenrain wenig anderes geschehen kann als Ballspiele. Die Anlage bietet wenig Möglichkeiten, sich auf dem Platz aufzuhalten, ohne aktiv oder passiv als Zuschauer am Spiel teilzunehmen. ${ }^{38}$

Die durch die Architektur hergestellte Anregung zielt primär auf spielerisch-sportliche Betätigung, die im Rahmen eines Mannschaftssports, also eines Sets von kodifizierten und gegebenen Regeln basiert. Dies mag auch die Doppelfunktion erklären, die der Sportplatz als Unterrichtsort und Ort von Nichtunterricht beinhaltet. Die Sozialisation zielt auf die Einübung von Regeln, Normen und rollenförmigem Handeln. Indem dies die dominante Disposition des Schulhofs ist, reproduzieren sich in gewissem Sinne die Bedeutungsstrukturen in der

38 Von Schülerinnen, die das Schulhaus Breitenrain besuchen, weiß ich, dass sie das Schulhaus und insbesondere den Pausenplatz nicht mögen. Durch die Platzgestaltung und mangels Alternativen sind sie zum Zuschauen der Spieler, vorwiegend Jungs, gezwungen. 
Adressierung der Schüler_innen, wie sie in der Rekonstruktion des Schulhauses expliziert wurde.

Sport hat in der Schule aber generell gesehen noch eine weitere Dimension. Die Schulgymnastik verkörpert fast prototypisch die Regulierung und Normierung der Körper durch die Machtdisziplin, wie Foucault (2006) sie unter einer machttheoretischen Perspektive sieht. Dabei dient die Disziplin nicht lediglich negativ dem Zugriff der Macht zwecks Machtdurchsetzung. Sie ist vor allem produktiv zu sehen. Sie produziert reale Wissensordnungen und damit einhergehende soziale Praktiken gerade auch im Blick auf den Umgang mit Sozialisanden: „Die Disziplin verfertigt Individuen: sie ist die spezifische Technik einer Macht, welche die Individuen sowohl als Objekte wie als Instrumente behandelt und einsetzt“" (Foucault 2012b: 199).

Architektur hat darin eine entscheidende und neue Aufgabe. Sie ist nicht mehr lediglich Repräsentation und Kontrolle, sondern eine formierende Kraft. Ihre Intention ist es, Verhaltensänderungen zu bewirken. Ziel der Macht ist „eine Architektur, die ein Instrument zur Transformation der Individuen ist: die auf diejenigen, welche sie verwahrt, einwirkt, ihr Verhalten beeinflussbar macht, die Wirkungen der Macht bis zu ihnen vordringen lässt, sie einer Erkenntnis aussetzt und sie verändert“ (Foucault 2012a: 222). Bei der Wirkung der Disziplinarmacht ist wesentlich, dass die Subjekte die Disziplin internalisieren, ohne dass die Disziplinarmacht sichtbar wird:

„Sie [die Disziplinarmacht] setzt sich durch, indem sie sich unsichtbar macht, während sie den von ihr Unterworfenen die Sichtbarkeit aufzwingt. In der Disziplin sind es die Untertanen, die gesehen werden müssen, die im Scheinwerferlicht stehen, damit die Zugriffe der Macht gesichert werden." (Foucault 2012a: 241)

Hier schließt sich die Argumentation. Denn ein zentrales Element der Sichtbarkeit von Macht, insbesondere was die militärische Macht betrifft, ist bei Foucault die Parade.

Foucault führt aus, dass diese nicht nur in Aktion sichtbar ist. Manifest kann dies zwar der Fall sein, latent ist für die Parade aber gerade bedeutsam, dass bereits die Platzgestaltung die Zeremonie einer Parade ausdrückt. Der leere Platz steht genauso für die Parade und die Machtdemonstration wie die Parade selbst. Diese Argumentation kann meiner Meinung nach auf Schulbauten übertragen werden. Die Errichtung von Schulhäusern, die als uniforme Schulkasernen, meist 
mit einem großen Rasenplatz, gebaut wurden, verkörpern eine Sinnstruktur, welche die Schule und den Schulraum in seiner Repräsentation und in seiner Funktion stark militärisch sieht und in welcher das Schulturnen in der Form öffentlich einsehbarer, paradehafter, uniformer Bewegungsabläufe wesentlich ist.

Kehren wir zum Schulgelände Breitenrain zurück. Am Beispiel des Pausenhofs wurde auf die Bedeutung des Sports sowohl aus einer intrinsisch sozialisatorischen Perspektive als auch aus einer disziplinierenden Perspektive hingewiesen. Zudem wurde eine räumliche Einheit von Schulturnen und Pausenhof festgehalten. Als nächste Sequenz soll daher die Turnhalle in den Blick gerückt werden.

Die Turnhalle manifestiert wie kein anderes Gebäude den Schulsport in Form des organisierten und angeleiteten Unterrichts. Die Erbauung der Turnhalle hat nicht nur funktional Auswirkungen auf Art und Weise des Schulsports. Sie läutet eine wesentliche Transformation des Schulareals ein.

Mit der L-förmigen Platzierung der Turnhalle bekommt der Schulhausplatz den Charakter eines Pausenhofs. Höfe sind sozial exklusive Räume. Ein Hof konstituiert einen zentralen sozialen Ort für die spezifische Gruppe, die Zugang zum Hof hat. Dabei gilt, je größer der Hof, umso größer ist in der Regel auch die soziale Gruppe, die Nutzungsrechte am Hof hat. Höfe konstituieren eine zentripetal ausgerichtete Praxis, die für exklusive, auf die soziale Gruppe bezogene Praktiken gedacht sind. Der Hof im betrachteten Schulbau zeichnet sich dadurch aus, dass er einen Hinterhof darstellt und es keinen direkten Zugang aus dem Gebäude in den Hof gibt. Er hat keine Durchgangsfunktion, sondern ist als Zone einer in sich geschlossenen Praxis konzipiert. Damit wird der Hof zu einem Annex des Gebäudes, also ein in sich relativ geschlossener und abschließbarer Funktionsraum. Der Schulhof manifestiert in seiner Abschottung einen Schutzoder Schonraum. Die Platzierung des Gebäudes hat zur Folge, dass die Schule dadurch einen stärkeren Binnenbezug erhält.

Welches ist also die spezifische Praxis, für dessen Sinnbild die Turnhalle steht? Welche Transformationen gehen im Schulturnen mit einer Turnhalle einher? Eine Turnhalle eignet sich ungleich weniger für die Massenveranstaltungen eines synchronen Turnens in der Schulgymnastik. Sie ermöglicht aber ganz neue Möglichkeiten von spezifischen Sportarten, sowohl von Mannschaftssport- als auch individuelle Sportarten. Eine Turnhalle bietet die Möglichkeit, Sportarten und -übungen spezifisch durchzuführen, wie sie nur in Kombination mit funktio- 
nalen Geräten und Infrastrukturen möglich wird. Eine Turnhalle beinhaltet ein Sammelsurium von Geräten, die unterschiedlichste Übungen an jeweils spezifischen Geräten erlauben. Es wird also ein Raum geschaffen, indem neue Möglichkeiten eröffnet werden. Diese sind vielfach an Artefakte gebunden, die jeweils ganz spezifische Gesten und Möglichkeiten beinhalten: Ringe laden zum Schwingen ein, ein Barren zum Barrenturnen, eine Kletterstange zum Klettern usw. Unter der Perspektive der Disziplinierung stellt eine Turnhalle einen ungleich vielfältigeren und feingliedrigeren Möglichkeitsraum dar, als dies ein Pausenplatz tut. Dabei sind die einzelnen Möglichkeiten durch die Artefakte zugleich funktional wesentlich eingeschränkter. Im Unterschied zur Gymnastik, in welcher der Körper mehr oder weniger selbstgenügsam sich seiner selbst bedient, können die Körperübungen und -techniken an spezifischen Geräten differenziert, verfeinert und perfektioniert werden.

Die Körperübungen sind voraussetzungsvoller. Wird die Elementargymnastik zum Beispiel bei Pestalozzi noch als Mittel der Sublimation von Aggression und Unzivilisiertheit betrachtet, also als psychisches Disziplinierungsprojekt (siehe Werder 1896), so setzen die sportlichen Tätigkeiten, die mit einer Turnhalle möglich werden, diese Selbstdisziplinierung bereits voraus. Die Einübung geht nicht mehr in einer Massenveranstaltung vonstatten, sondern beinhaltet individuelle Übungen. Die Gymnastik stellt ein Bewegungsprinzip dar, in der Restriktionen im Prinzip durch den eigenen Körper ausgedrückt werden. Die Turnhalle steht für ein Set an Artefakten, das diese Limitierung außer Kraft setzt. Der Möglichkeitsraum wird um ein Vielfaches erweitert.

Da die Turnhalle Praktiken erlaubt, die mithilfe von Artefakten die Limitierungen durch die menschlichen Körper außer Kraft setzen, wird eine Disziplinierung von Körperkontrolle, aber auch in der Arbeitsweise bereits vorausgesetzt. An Geräten kann nicht oder nur sehr bedingt synchron geturnt werden. Ein Turnunterricht an unterschiedlichen Geräten muss an verschiedenen Etappen binnendifferenziert durchgeführt werden. Es beinhaltet eine Ausdifferenzierung von Unterricht. Und es bedingt, dass die Schüler_innen die Übungen zumindest teilweise selbsttätig durchführen.

Von Interesse ist, dass der Bau der Turnhalle einen Umgang mit dem Schulsport ableiten lässt, der sich auch in der Umgebung feststellen lässt. Die große Mehrheit der Wohnhäuser in der Umgebung stammt aus derselben Zeit, in der auch die Turnhalle erstellt wurde. Im Gegensatz zu den Arbeiter- und Kleinge- 
werbehäusern im Norden des Bildausschnittes hat sich mit den Wohnhäusern und der damit gekoppelten Lebensweise von Lohnarbeit, einem Status als Angestellte, auch eine größere Disziplinierung des Lebenswandels realisiert. Dies betrifft sowohl die Körperkontrolle als auch die psychosozial-mentale Disziplinierung, die eine stärkere Internalisierung von diszipliniertem (Rollen-)Handeln erfordern, als dies noch bei proletarischen Schichten in der Mitte des 19. Jahrhunderts der Fall war.

\subsubsection{Schule und Körperhygiene - Tilgung des Individuellen}

Ein Merkmal, das die Schule Breitenrain in ihrer heutigen Ausgestaltung ganz wesentlich geprägt hat, ist der Bau der Garderobe, die eine Neugestaltung des Schulhausareals nach sich zog. Die Platzierung mitten in den Schulhof wirkt brutal, die Großzügigkeit und die Binnenzentrierung des Ensembles durch den Hof wird zerstört. Damit geht auch das gemeinschaftliche Moment verloren, welches den Hof originär ausgezeichnet hat. Der Hof wird durch den Bau der Garderobe in verschiedene Räume fragmentiert. Abgesehen vom Basketballfeld bleibt ein kleiner Raum zwischen den Gebäuden und einige Nischen hinter dem Annex, welche als versteckte Räume übrig bleiben. Zudem hat die Raumvernichtung den Effekt, dass das Schulareal über die Straße hinaus erweitert werden muss.

Die Garderobe ist als Annex an die Turnhalle angedockt. Das hergestellte Raumvolumen scheint im Verhältnis zum Platzbedarf und im Verhältnis der urbanen Umgebung sehr gering. Die Auswirkungen auf das Gebäudeensemble sind im Verhältnis zum Raumgewinn enorm. Es sind Varianten denkbar, wie eine Garderobe in das Ensemble hätte integriert werden können, die einen weniger drastischen Wandel und einen wesentlich sparsameren Umgang mit dem Raum erlaubt hätten. Der Bau einer Garderobe in einer postmodernen Bauweise wird eine Bedeutsamkeit zugestanden, welche eine funktionale und ästhetische Destruktion eines ausgeglichenen Gefüges rechtfertigt oder diese Destruktion gar bewusst zum Inhalt hat.

Rein funktional ist eine Garderobe ein Raum, in dem man sich in der Regel so kurz wie möglich aufhält und der die meiste Zeit leer steht. Garderoben sind Durchgangsräume, in denen ein sichtbarer Statuswechsel vonstattengeht und in 
denen persönliche Alltagsgegenstände deponiert werden: Es sind Aufbewahrungsorte für die Kleidung und andere persönliche Gegenstände. Zudem sind in den Gardeoben die Duschen untergebracht. ${ }^{39}$ Garderoben sind untrennbar mit Körperhygiene und mit den gesellschaftlichen Vorstellungen darüber verbunden.

Garderoben haben überdies die Funktion einer Statusveränderung. Sie können als eine Art Pufferzone betrachtet werden. In der Schulgarderobe werden auch die alltäglichen außerschulischen, also persönlichen Gegenstände temporär aufbewahrt. Wenn Kleidung als zweite Haut betrachtet wird, beinhalten deren Schichten unterschiedliche Nähe- und Intimitätsbereiche. In dieser Überlegung sind Schulgarderoben dafür da, die äußersten Schichten aufzubewahren, die Auskunft über Status, Geschlecht, Klassenzugehörigkeit usw. geben. Mit dem Ablegen der Kleiderschichten geht auch ein impliziter Statusübergang einher.

Das Ablegen der Jacken durch die Schüler_innen ist auch eine Art von Übergangsritual zum Unterricht. Dies ist in Ländern mit Schuluniformen anders geregelt. Dort sind die Schüler_innen schon im öffentlichen Raum als Schüler_innen quasi markiert. Da dies in der Schweiz nicht der Fall ist, bekommen die Garderoben die Funktion einer Status-Schranke. Indem die privaten Gegenstände und die Kleider, die für die Öffentlichkeit bestimmt sind, abgelegt werden, wird die Rolle der Schülerin bzw. des Schülers angenommen. Die Lage der Garderoben gibt Auskunft darüber, wo innere Schranken der Innen-AußenAbgrenzung liegen, die mit Statusübergängen einhergehen. Oder anders gesagt, wie Übergänge zum Unterrichtsort gestaltet werden.

Für die Turngarderobe gilt die Statusveränderung nun noch gesteigert, da die intimsten Kleiderschichten mitbetroffen sind. Für den Turnunterricht werden die alltäglichen und die meisten persönlichen Kleidungsstücke abgelegt. Noch expliziter steht die Dusche für eine Tilgung des Individuellen. Damit sind die noch intimeren Bereiche betroffen. Der eigene Körper wird durch die Nacktheit sichtbar. Die Duschen dienen dazu, die körpereigenen Pheromone, also den individuellen Geruch, abzuwaschen. Der Geruch, der, wie es der Volksmund treffend ausdrückt, darüber bestimmt, ob man jemanden riechen kann, wird getilgt. Auffällig ist hier, dass die Garderoben als offene Bereiche konzipiert sind. Es gibt keine Privatsphäre in Form von unterteilten Duschen, es sind keine ,Scham- 
wände` eingezogen. Die Reinigung vom Individuellen bekommt so als rituelle Reinigung in der Gruppe einen zusätzlichen Charakter: Die gemeinsame Dusche in der Halböffentlichkeit der Peer Group dient nicht nur der physischen Reinigung von Schmutz, sondern zugleich einer psychogenen Reinigung vom ,Individuellen'. Für das moderne Subjekt ist die Elimination der Pheromone als individuelle Duftmarke und deren Ersetzung durch parfümierte Produkte relativ zentral. Mehr noch, sie wird fast Bedingung für rein rollenförmige Beziehungen, in denen die individuierte Person in den Hintergrund rückt.

\subsubsection{Entgrenzung der Schule}

Mit dem Bau der Garderobe geht die explizierte Verschiebung der Innen-AußenAbgrenzung in den gegenüberliegenden Park und die erzwungene Neugestaltung des Schulhofs einher. Das Augenmerk ist vorerst auf die Ein- und Abgrenzung der Schule und deren Entgrenzung zu richten. Was die Schulanlage Breitenrain besonders auszeichnet, ist, dass die Einfriedung nordseitig aufgehoben ist. Die Absenz der Einfriedung im konkreten Fall verweist umso deutlicher auf deren Funktion. Denn Ein- oder Umfriedungen sind funktional mit Fragen der Exklusivität und damit der In- und Exklusion verbunden. Sie stecken ein ,privates ' Territorium ab, auf welches ein claim erhoben wird. Sehr oft sind auch Regeln der Mitgliedschaft mit Schranken verbunden, die zugleich mit Rollenübernahmen einhergehen. Mit dem Durchschreiten der Einfriedung wird eine Rolle übernommen: Das Kind wird zum Schüler, zur Schülerin, der Vorübergehende zum Besucher.

Beim Schulhaus Breitenrain sind die Verhältnisse unklar, da Zwischenformen von halb öffentlichem Raum im Bereich der Erschließungen vorhanden sind: Die überbreiten Gehsteige und Blumenbeete lassen eine Art von Pufferzone oder ein System mehrerer Schranken entstehen. Das Durchschreiten der äußeren Einfriedung ist ein Betreten des privaten Bereichs. Das eintretende Subjekt wird identifiziert, also prädiziert, und darauf folgt auch eine Adressierung, indem ihm unabhängig von der subjektiven Intention eine Rolle zugewiesen wird. Mit dem Schritt durch das Schultor oder durch die angedeutete Schranke der Umfriedung tritt ein Subjekt automatisch aus der Anonymität eines Unbestimmten heraus. Es wird in einer Rolle prädiziert als Schülerin/Schüler, als Besucher oder auch als 
Eindringling und Störenfried, wenn das Betreten nicht legitimiert ist. Die Kinder werden mit dem Eintritt zu Schüler_innen, wenn auch noch in einem vergleichsweise informellen Status. Denn der Pausenplatz ist das Territorium, das den Schüler_innen zur autonomen Aneignung mehr oder weniger überlassen ist. Es sind Dinge erlaubt oder gar erwünscht, die innerhalb der Gebäude nicht toleriert werden: Das Spielfeld stellt geradezu die Intention her zu rennen, während dies in den Schulgebäuden in der Regel untersagt ist.

Die Einfriedung markiert nicht nur eine Grenze von Inklusionsregeln, sondern auch diejenige von Zuständigkeiten. Dies gilt auch rechtlich. So sind außerhalb der Einfriedung die Eltern für ihre Kinder zuständig und haften auch für sie, während die Schulen die Obhuts- und Aufsichtspflicht auf dem Schulgelände innehaben. Am Schulhaus Breitenrain ist festzustellen, dass diese trennscharfe Markierung aufgehoben ist. Der Schulraum ragt in den öffentlichen Raum hinein: einerseits auf die Straße, die mit blauen Linien markiert wird, und andererseits über die Straße hinaus in den öffentlichen Park hinein. Dabei geht die Erweiterung nicht mit einem Exklusivitätsanspruch einher. Sowohl die Straße als auch Spielplatz und Park werden in ihren öffentlichen Funktionen genutzt. Die Schule beansprucht eine Mitbenutzung öffentlicher Infrastruktur. Damit wird die Innen-Außen-Abgrenzung unscharf und eindimensional geöffnet. Auf dem Gebiet der Straße und des Parks fallen öffentlicher Raum und Schulraum zusammen. Die Entgrenzung hat zur Folge, dass die Schule einen Raum beansprucht, für den sie nicht exklusiv zuständig ist, was wiederum Auswirkungen auf die Obhutspflicht der Schule hat. Auch diese ist nun räumlich nicht mehr trennscharf bestimmt und muss erst hergestellt werden.

Die Entgrenzung kann aber auch die umgekehrte Richtung annehmen: Es manifestiert sich ein Geltungsbereich, der über den Perimeter hinaus in Anspruch genommen werden kann. Die Schule reklamiert eine Zuständigkeit nicht mehr ausschließlich in ihrem abgeschotteten Bereich, sie weitet ihre Zuständigkeit aus. Die Schule stellt sich damit indirekt auch als Institution dar, die nicht einen eingegrenzten Bereich in der Sozialisation der Schüler_innen umfasst, der komplementär zur familialen Sozialisation steht.

Hier reproduziert sich eine Sinnstruktur, die uns in der Interpretation schon mehrmals begegnet ist. Unterricht und Nichtunterricht verwischen zusehends. Die Gestaltung der Räume, die für den Nichtunterricht gedacht sind, wird genauso mit pädagogischen Intentionen besetzt wie die der Klassenzimmer. 


\subsection{Fazit - Performativ-disponierende Architektur zur Erzeugung disziplinierter und subalterner Subjekte}

Die Analyse des Schulhauses Breitenrain fördert eine Bedeutungsstruktur zutage, die ihre Wurzeln in einer militärischen Struktur haben und in welcher die Internalisierung von Normen und Wissen über Körpertechniken das maßgebliche sozialisatorische Element darstellen. Auch gegen aussen repräsentiert das Schulhaus das Militär als maßgebliche schulische Erziehungsinstanz. ${ }^{40}$ Das Gebäude zielt in seiner Repräsentation und Funktionalität auf ein gemeinschaftliches Ganzes, indem die Individualität getilgt, diese durch Uniformität ersetzt und die Subjekte dem Staat ein- und untergeordnet werden.

Der (Schul-)Sport ist das ästhetisch und räumlich auffälligste Element des Schulareals. Darin manifestieren sich Bedeutungsstrukturen, welche die Pädagogik als stark militärisch konnotiert darlegen: Disziplin, Kollektivität, Rollenverhalten und eine Raumorganisation, die keine Räume für Privatheit oder Anregungen zu autonomen Tätigkeiten herstellen. Die Anregungen, die durch die Architektur hergestellt werden, liegen in einer pädagogischen Intention, die Sozialisation vor allem über Bewegung, über Körper- und Leiberfahrung repräsentiert.

Der (Schul-)Sport ist omnipräsent sowohl als organisierte schulische Bildungsveranstaltung als auch über die durch Architektur hergestellten Anregungen des Schulareals. Die Architektur produziert im Wesentlichen Handlungsdispositionen, die auf eine leibzentrierte Sozialisation gerichtet sind, die über Körper- und Leiberfahrung gesellschaftliche Regeln, Rollenhandeln, aber auch Selbst- und Körperkontrolle trainieren und inkorporieren.

Dabei wurde eine Transformation in der Art und Bedeutung der körperbasierten Pädagogik rekonstruiert. Von einer synchronen Massenveranstaltung in der Schulgymnastik, die vor allem auf die Herstellung eines uniformen, disziplinierten Subjekts gerichtet ist, zu einer intrinsisch angestoßenen Sozialisation

40 Zeitgleich mit dem Bau des Breitenrain-Schulhauses veranlasst der Bund 1868, dass die Kantone allen Knaben einen auf militärische Aspekte ausgelegten Turnunterricht zu erteilen haben (Giuliani 2001: 481 f.). 1874 wird der Turnunterricht als Teil der Landesverteidigung definiert und unter die Obhut des Eidgenössischen Militärdepartementes gestellt, was eine Anomalie im subsidiär organisierten schweizerischen Bildungssystem ist. 
über äußere, an Objekte gebundene Verhaltensaufforderungen. Oder anders gesagt: Der Sport wird von einer von außen initiierten, koordinierten und überwachten Disziplinierung im Schulturnen zu einer inneren Aneignung eines selbstdisziplinierenden Moments, das sowohl als Antipode geistiger Bildung als auch als (Wieder-)Herstellung der geistigen Leistungsbereitschaft für einen kognitiv ausgerichteten Unterricht rekonstruiert werden kann. Dieses Muster reproduziert sich zahlreich.

Die Öffnung des Schulraums zu einem Freizeitraum findet eine Entsprechung in einer räumlichen und symbolischen Entgrenzung der Schule. Die pädagogisch intendierte Architektur stellt auch in Räumen und Zeiten des Nichtunterrichts Anregungen her, die Einfluss auf die Tätigkeiten der Sozialisanden nehmen und diese vorstrukturieren. Dabei sind die Bedeutungsstrukturen, die in der Architektur vorliegen, sinnaffin mit derjenigen des Unterrichtes.

Auch im Nichtunterricht werden durch die wiederholten Bewegungen nicht nur Körpertechniken wie der spezifische Körpergebrauch zum Sitzen, Schreiben oder zum Fußballspielen geübt, es wird zugleich immer auch ein gesellschaftliches Wissen internalisiert. Dies beinhaltet vor allem implizites Wissen in der Internalisierung gesellschaftlicher Regeln und Normen. Man kann sagen, die Architektur der Schulanlage ist im Wesentlichen darauf gerichtet, das Wilde und Ungestüme des kindlichen, ungeformten Körpers in einen durch sportliche Betätigung sozialisierten und habitualisierten Körper zu transformieren.

Die rekonstruierte Raumaufteilung zwischen leiblicher und geistiger Bildung muss als konstitutiv für die öffentlich-rechtliche Schule ausgelegt werden. Dabei wurde dargelegt, wie sich die leibliche Disziplinierung wesentlich in zwei Achsen transformiert hat: einerseits vom gleichförmigen, in einer Masse vollzogenen, aber auf den eigenen Körper ausgerichteten Turnen zu einem individuellen Agieren in einem Team, was die Antizipation des eigenen Rollenhandelns bedingt. Andererseits ist es eine (Rück-)Transformation von Inhalten des angeleiteten Unterrichts in der Turnstunde zu selbsttätigem Handeln in den Zeiten des Nichtunterrichts. Damit sind nicht nur die Pausen, sondern auch die Freizeit gemeint, was eine Ausweitung des Geltungsanspruchs der Schule darstellt. Dies manifestiert sich im Sportplatz, der als azurblaue Lagune auch symbolisch markiert wird. Der Sport manifestiert damit eine Wandlung vom disziplinierenden Vorunterricht in der Schulgymnastik mit dem Ziel der Herstellung der Wehr- 
tüchtigkeit zu einer Funktion der Wiederherstellung der geistigen Leistungsbereitschaft. Oder anders gesagt: als Teil sogenannter Work-Life-Balance.

In einem gewissen Sinne reproduziert sich diese Sinnstruktur, wenn der Blick über die Straße auf den Spielplatz gerichtet wird, der als erweiterter Teil des Schulareals rekonstruiert wurde. Der Spielplatz wurde vor allem unter dem Aspekt der räumlichen Ausdehnung, der Verschiebung und stellenweisen Aufhebung der Innen-Außen-Abgrenzung betrachtet. In seiner Funktion ist es ein Spielplatz. Es ist ein räumliches Arrangement, in welchem zahlreiche Spielgeräte angebracht sind, die für Kinder gebaut und als anregende Umgebung für diese gedacht sind. Der Spielplatz ist eine strukturierte Umgebung, eine sogenannte Lern- und Bildungslandschaft, die aus zahlreichen Installationen besteht, die für unterschiedliche Praktiken vorgesehen sind. Es sind Installationen, welche die Kinder zu autonomen und selbsttätigen Praktiken animieren: Klettern, Buddeln, Schaukeln, Rutschen. In der Regel sind es monofunktional ausgerichtete Installationen (ausführlich im Kapitel 7.4.1).

Im Weiteren zeigt sich nicht nur in Bezug auf den Umgang mit dem eigenen Körper eine Bedeutungsstruktur, welche disziplinierende Momente nach ,innen verlegt. Die Innen-Außen-Abgrenzung und die Übergänge werden zusehends fließend. Die ehemals ,versteinerte "Einfriedung wird ersetzt durch ,einverleibte", habitualisierte Regeln, die die Nutzungsrechte regeln. Aber auch die sitzende Lernhaltung und die spezifisch schulische Klassenanordnung, die früher ziemlich brachial durch die Subsellien erzwungen wurden, sind heute in Form von Regelwissen, das an Gegenstände gekoppelt ist, internalisiert.

Diese Entgrenzung zwischen Unterricht und Nichtunterricht findet sich als Entgrenzung von Schule und Nichtschule auch im Verhältnis des Schulhauses Breitenrain zu seiner Nachbarschaft. Mit der Erweiterung des Schulareals als Überlappung in den Stadtteilpark wird dieser von der Schule mitbesetzt. Damit vollzieht sich räumlich, was dem Anspruch der Schule längst inhärent ist: die Ausweitung des Geltungsbereichs der Schule auf alle Lebensbereiche.

Architektur ist dauerhafter und wesentlicher Teil des gesellschaftlichen Gedächtnisses. Mit dem Schulhaus Breitenrain wurde bewusst ein Schulhaus gewählt, das aus einer Zeit stammt, wo sich das öffentlich-rechtliche Schulsystem konsolidierte. Aus der Architekturanalyse konnte rekonstruiert werden, dass die Schule Mittel und Teil einer flächendeckenden Institutionalisierung einer neuen 
Form staatlicher Repräsentation ist, die nicht lediglich ein Symbolbild repräsentiert, sondern im Wesentlichen eine moderne Form des Regierens darstellt.

Das Schulhaus Breitenrain steht exemplarisch für einen neuen Gebäudetyp Schule, der nicht mehr als Behälterraum konzipiert ist. Das Ziel ist nicht, ein Dach über dem Kopf zu haben und einen Raum zu schaffen, in dem plurale Unterrichtsformen Platz finden, wie dies noch in Pestalozzis Schulwohnstube der Fall war (vgl. Kap. 7.3). Das Haus repräsentiert in Aussehen und Funktionalität eine neue Form des Zugriffs auf die Schüler_innen über Architektur. Die Architektur ist handlungsleitend und disziplinierend, lässt keinen Raum für persönliche Autonomie und adressiert keine Individuen, sondern eine Gruppe uniformer Schüler_innen. Das Schulhaus ist in dem Sinne nicht nur Grundausstattung für den pädagogischen Unterricht, sondern gleichzeitig eine Arena des Regierens durch Artefakte: Dinge werden so platziert, dass sie das Verhalten beeinflussen. Architektur bekommt eine neue Aufgabe. Sie ist nicht mehr lediglich Repräsentation und Kontrolle, sie wird zu einer formierenden Kraft, die darauf abzielt, Verhaltensänderungen zu bewirken.

Wenn die Architektur des Schulhauses Breitenrain als Repräsentation der Funktionalität gelesen wird, dann liegt die Funktionalität der Schule primär in der Disziplinierung, in der Tilgung von Individuellem und der Schaffung von gehorsamen Subjekten. In der Architektur des Schulhaus Breitenrain manifestiert sich die Sozialisationsfunktion der Schule vor allem in einem disziplinierenden Modus, indem dieser auf einen disziplinierten, nach gesellschaftlichen Regeln verlaufenden Umgang mit dem Körper gerichtet ist.

Als Nächstes wird ein Fall betrachtet, der eine formative Architektur herzustellen verspricht, welche die zeitdiagnostischen Deutungen über Schule, Sozialisation und Bildung verkörpert.

Open Access Dieses Kapitel wird unter der Creative Commons Namensnennung 4.0 International Lizenz (http://creativecommons.org/licenses/by/4.0/deed.de) veröffentlicht, welche die Nutzung, Vervielfältigung, Bearbeitung, Verbreitung und Wiedergabe in jeglichem Medium und Format erlaubt, sofern Sie den/die ursprünglichen Autor(en) und die Quelle ordnungsgemäß nennen, einen Link zur Creative Commons Lizenz beifügen und angeben, ob Änderungen vorgenommen wurden.

Die in diesem Kapitel enthaltenen Bilder und sonstiges Drittmaterial unterliegen ebenfalls der genannten Creative Commons Lizenz, sofern sich aus der Abbildungslegende nichts anderes ergibt. Sofern das betreffende Material nicht unter der genannten Creative Commons Lizenz steht und die betreffende Handlung nicht nach gesetzlichen Vorschriften erlaubt ist, ist für die oben aufgeführten Weiterverwendungen des Materials die Einwilligung des jeweiligen Rechteinhabers einzuholen.

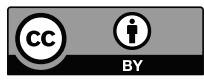

